TITLE:

\title{
Symmetry Parameter Constraints from a Lower Bound on Neutron- matter Energy
}

\section{$\operatorname{AUTHOR}(S)$ :}

Tews, Ingo; Lattimer, James M.; Ohnishi, Akira; Kolomeitsev, Evgeni E.

\section{CITATION:}

Tews, Ingo ...[et al]. Symmetry Parameter Constraints from a Lower Bound on Neutron-matter Energy. The Astrophysical Journal 2017, 848(2): 105.

ISSUE DATE:

2017-10-20

URL:

http://hdl.handle.net/2433/233603

RIGHT:

(C) 2017. The American Astronomical Society. All rights reserved. 


\title{
Symmetry Parameter Constraints from a Lower Bound on Neutron-matter Energy
}

\author{
Ingo Tews ${ }^{1,2}$ (1), James M. Lattimer ${ }^{3}$ (1) , Akira Ohnishi $^{4}$ (1), and Evgeni E. Kolomeitsev ${ }^{5,6}$ (1) \\ Institute for Nuclear Theory, University of Washington, Seattle, WA 98195-1550, USA; itews@uw.edu \\ 2 JINA-CEE, Michigan State University, East Lansing, MI 48823, USA \\ 3 Department of Physics and Astronomy, Stony Brook University, Stony Brook, NY 11794-3800, USA; james.lattimer@stonybrook.edu \\ ${ }^{4}$ Yukawa Institute for Theoretical Physics, Kyoto University, Kyoto 606-8502, Japan; ohnishi@yukawa.kyoto-u.ac.jp \\ 5 Faculty of Natural Sciences, Matej Bel University, Tajovskeho 40, SK-97401 Banska Bystrica, Slovakia; e.kolomeitsev@gsi.de \\ ${ }^{6}$ Joint Institute for Nuclear Research, RU-141980 Dubna, Moscow Region, Russia \\ Received 2017 June 30; revised 2017 September 6; accepted 2017 September 17; published 2017 October 20
}

\begin{abstract}
We propose the existence of a lower bound on the energy of pure neutron matter (PNM) on the basis of unitary-gas considerations. We discuss its justification from experimental studies of cold atoms as well as from theoretical studies of neutron matter. We demonstrate that this bound results in limits to the density-dependent symmetry energy, which is the difference between the energies of symmetric nuclear matter and PNM. In particular, this bound leads to a lower limit to the volume symmetry energy parameter $S_{0}$. In addition, for assumed values of $S_{0}$ above this minimum, this bound implies both upper and lower limits to the symmetry energy slope parameter $L$, which describes the lowest-order density dependence of the symmetry energy. A lower bound on neutron-matter incompressibility is also obtained. These bounds are found to be consistent with both recent calculations of the energies of PNM and constraints from nuclear experiments. Our results are significant because several equations of state that are currently used in astrophysical simulations of supernovae and neutron star mergers, as well as in nuclear physics simulations of heavy-ion collisions, have symmetry energy parameters that violate these bounds. Furthermore, below the nuclear saturation density, the bound on neutron-matter energies leads to a lower limit to the density-dependent symmetry energy, which leads to upper limits to the nuclear surface symmetry parameter and the neutron-star crust-core boundary. We also obtain a lower limit to the neutron-skin thicknesses of neutronrich nuclei. Above the nuclear saturation density, the bound on neutron-matter energies also leads to an upper limit to the symmetry energy, with implications for neutron-star cooling via the direct Urca process.
\end{abstract}

Key words: dense matter - equation of state - stars: neutron

\section{Introduction}

The nuclear symmetry energy $(S(u))$ is one of the decisive ingredients in compact-star astrophysics as well as in nuclear physics. It provides the pressure of neutron-star matter, which is nearly pure neutron matter (PNM) near the saturation density $n_{0} \simeq 0.16 \mathrm{fm}^{-3}$, and largely determines neutron-star radii (Lattimer \& Prakash 2001) and therefore properties of their crusts, moments of inertia, tidal polarizabilities, and binding energies (Lattimer \& Prakash 2007). The symmetry energy is also important in calculations of the $r$-process (Mumpower et al. 2016), supernovae (Fischer et al. 2014), and neutron-star mergers (Bauswein et al. 2016). Terrestrial experiments measuring nuclear masses, dipole resonances, and neutronskin thicknesses can constrain the symmetry energy (Lattimer \& Lim 2013), as can experiments using normal and radioactive nuclear beams (Oertel et al. 2017).

The symmetry energy can be obtained from the energy per particle in nuclear matter, $E(u, x)$, where $x$ is the proton fraction and $u=n / n_{0}$ is the density in units of saturation density $n_{0}$. The energy per particle at a given density varies between a minimum, symmetric nuclear matter (SNM, $x=0.5$ ), and a maximum, PNM $(x=0)$. The symmetry energy is defined as the difference of these energies:

$$
S(u)=E(u, 0)-E(u, 1 / 2) .
$$

One can expand the $E(u, x)$ in terms of the neutron excess $(1-2 x)$. From the symmetry properties of nuclear matter, there are no terms with odd powers of the neutron excess, so the lowest-order term in the expansion is quadratic, i.e.,

$$
\begin{aligned}
E(u, x)= & E(u, 1 / 2)+S_{2}(u)(1-2 x)^{2} \\
& +S_{4}(u)(1-2 x)^{4}+\ldots .
\end{aligned}
$$

There is little experimental evidence concerning the magnitude of quartic and higher-order terms because laboratory nuclei are nearly symmetric. Studies suggest that these terms are small (Carbone et al. 2014; Wellenhofer et al. 2016). If the energy is divided into kinetic and potential contributions, then the kinetic energy of degenerate noninteracting fermions can be expanded as

$$
\begin{gathered}
E_{\mathrm{kin}}(u, x)=\frac{3}{5} \frac{\hbar^{2}}{2 m}\left(3 \pi^{2} n_{0} u\right)^{2 / 3}\left[(1-x)^{5 / 3}+x^{5 / 3}\right] \\
=T\left[1+\frac{5}{9}(1-2 x)^{2}+\frac{5}{243}(1-2 x)^{4}+\cdots\right],
\end{gathered}
$$

where $T=3 \hbar^{2}\left(3 \pi^{2} n_{0}\right)^{2 / 3} /\left(2^{2 / 3} 10 m\right) \simeq 22.1 \mathrm{MeV}$. Keeping instead only the quadratic term in Equation (2), one would obtain

$$
E_{\text {kin,quad }}=T\left[1+\left(2^{2 / 3}-1\right)(1-2 x)^{2}\right],
$$

so the kinetic contributions to the symmetry energy to quadratic order would differ by

$$
\Delta S_{\text {kin }}=S_{\text {kin }, \text { quad }}-S_{\text {kin }}=T\left[2^{2 / 3}-\frac{14}{9}\right] \simeq 0.032 T .
$$


This difference would have to be made up from quartic and higher-order terms. Indeed, the quartic contribution to the kinetic symmetry energy contributes $5 T / 243=0.021 T$, about $2 / 3$ of the missing amount.

Most theoretical calculations of the potential contribution to the symmetry energy also find only small corrections beyond the quadratic term (Carbone et al. 2014; Wellenhofer et al. 2016). Recent calculations of neutron-rich matter (Drischler et al. 2016b) have shown that up to densities approaching $u \sim 1.5$ the quadratic assumption is accurate to better than $1 \mathrm{MeV}$ in the symmetry energy per particle for all values of $x$. However, note that the kinetic contributions to quartic and higher-order terms vary as $u^{2 / 3}$ and potential contributions vary with higher powers of $u$. Thus when $u \gg 1$, the neglect of these terms may be unjustified.

Keeping only the quadratic term for the entire range of proton fractions, i.e., assuming $S(u)=S_{2}(u)$, the energy and pressure of PNM can be expressed through the traditional symmetry energy parameters

$$
\begin{gathered}
S_{0}=\frac{1}{8}\left(\frac{\partial^{2} E}{\partial x^{2}}\right)_{u=1}=(S)_{u=1}, \\
L=\frac{3}{8}\left(u \frac{\partial^{3} E}{\partial u \partial x^{2}}\right)_{u=1}=3\left(u \frac{d S}{d u}\right)_{u=1}, \\
K_{\text {sym }}=\frac{9}{8}\left(u^{2} \frac{\partial^{4} E}{\partial u^{2} \partial x^{2}}\right)_{u=1}=9\left(u^{2} \frac{d^{2} S}{d u^{2}}\right)_{u=1},
\end{gathered}
$$

and

$$
Q_{\mathrm{sym}}=\frac{27}{8}\left(u^{3} \frac{\partial^{5} E}{\partial u^{3} \partial x^{2}}\right)_{u=1}=27\left(u^{3} \frac{d^{3} S}{d u^{3}}\right)_{u=1} .
$$

The symmetry energy can then be Taylor expanded around the saturation density, $u=1$,

$$
\begin{aligned}
S(u)= & S_{0}+\frac{L}{3}(u-1)+\frac{K_{\text {sym }}}{18}(u-1)^{2}+\frac{Q_{\text {sym }}}{162}(u-1)^{3} \\
& +\mathcal{O}\left[(u-1)^{4}\right] .
\end{aligned}
$$

The most important parameters are $S_{0}$ and $L$, the symmetry energy, and its slope at $u=1 . K_{\mathrm{sym}}$ and $Q_{\mathrm{sym}}$ are the incompressibility and skewness parameters, respectively.

An abundance of information concerning the allowed ranges for $S_{0}$ and $L$ exists from nuclear experiments and theoretical studies of neutron matter. In particular, a strong positive correlation between their values can be deduced in a nearly model-independent manner from nuclear binding energies (Lattimer \& Lim 2013). $K_{\text {sym }}$ describes the curvature of $S$ near $u=1$ and can be probed in a limited way using the giant monopole resonance; see Blaizot (1980), Farine et al. (1997), and Stone et al. (2015). $Q_{\text {sym }}$ describes the skewness of $S$ near $u=1$, but it is extremely difficult to infer its value from experimental data. Thus, experimental and theoretical knowledge of $K_{\text {sym }}$ and $Q_{\text {sym }}$ is poor.

With knowledge of the binding energy of SNM at saturation, $E_{0} \simeq-16 \pm 0.5 \mathrm{MeV}, S_{0}$ determines the energy per particle of PNM at the same density: $E(1,0)=S_{0}+E_{0}$. In addition, the pressure $p(u, x)$ of PNM is directly related to $L: p(1,0)=$ $L n_{0} / 3$. PNM is a system of relevance for astrophysics because neutron-star matter is very close to PNM due to the small proton fractions in $\beta$-equilibrium near the nuclear saturation density. There have been many microscopical determinations of the neutron-matter equation of state, using a multitude of nuclear Hamiltonians and many-body methods. These include, e.g., self-consistent Green's function (SCGF) methods (see Drischler et al. 2016a for recent results), Quantum Monte Carlo (QMC) calculations (Gezerlis \& Carlson 2010; Gandolfi et al. 2012; Lynn et al. 2016), many-body perturbation theory (MBPT) calculations (Hebeler \& Schwenk 2010; Tews et al. 2013), and variational methods (Friedman \& Pandharipande 1981; Akmal et al. 1998; Togashi \& Takano 2013). Based on these calculations one can obtain constraints on the symmetry energy parameters if one assumes the quadratic expansion to be approximately valid. The extracted symmetry energy parameters are consistent with experimental determinations (Lattimer \& Lim 2013; Tews et al. 2013). Due to the intimate connection of PNM with the symmetry energy, any additional information about PNM translates into additional constraints on $S_{0}$ and $L$. Establishing a lower limit for the energy of PNM, e.g., would result in general limits to the symmetry energy parameters that so far have not been considered.

One possiblilty of a general lower limit could come from the unitary gas (UG). Universal behavior emerges for fermions interacting via pairwise $s$-wave interactions with an infinite scattering length $\left(a_{s}\right)$ and a vanishing effective range $\left(r_{\text {eff }}\right)$, i.e., the UG (see (Zwierlein 2015) for an historical review). Since in this case the average particle distance is the only length scale of the system, the ground state energy per particle in the UG, $E_{\mathrm{UG}}$, is proportional to the Fermi energy $E_{\mathrm{F}}, E_{\mathrm{UG}}=\frac{3}{5} E_{\mathrm{F}} \xi_{0}$, where the Bertsch parameter $\xi_{0}$ has the experimentally measured value of $\xi_{0} \simeq 0.37$ (Ku et al. 2012; Zürn et al. 2013).

PNM at very low densities, e.g., $n \sim 0.01 n_{0}$, where only $s$-wave contributions are important, is considered to show crossover behavior (Matsuo 2006) and to be close to the unitary limit since the $s$-wave scattering length of the $n n$ system is $a_{s}=-18.9 \mathrm{fm}$ (Machleidt \& Slaus 2001). This corresponds to $\left(a_{s} k_{\mathrm{F}}\right)^{-1} \simeq-0.15(-0.025)$ at $0.01 n_{0}\left(2 n_{0}\right)$, where $k_{\mathrm{F}}=\left(3 \pi^{2} n\right)^{1 / 3}$ is the neutron Fermi momentum, whereas the UG limit is $\left(a_{s} k_{\mathrm{F}}\right)^{-1}=0$. However, at large densities, differences in the underlying interactions become important and lead to different effects in both systems. This includes effective-range effects, interactions in higher partial waves, tensor contributions, etc. As an example, while the UG has a vanishing effective range, neutrons have an effective range of $r_{\text {eff }} \sim 2.7 \mathrm{fm}$ (Machleidt \& Slaus 2001).

While it has been suggested before that the UG is lower in energy than PNM, e.g., Carlson et al. (2002, 2012) and Gandolfi et al. (2015), it has so far not been applied to obtain limits on the symmetry energy parameters. We therefore follow the implications of making that conjecture,

$$
E_{\mathrm{PNM}}(n) \geqslant \frac{3}{5} \xi_{0} E_{F}(n)
$$

at densities of $n \lesssim 1.5 n_{0}$. We demonstrate that the nuclear symmetry energy parameters are thereby significantly constrained by the UG energy.

\section{The UG as a Lower Energy Limit to Pure Neutron Matter}

Nucleon-nucleon (NN) scattering can be described in terms of phase shifts in different partial waves. A positive phase shift in a particular scattering channel indicates attractive 
interactions, while a negative phase shift signals repulsive interactions in that channel. For neutrons at low energies, Schwinger (1947) showed that the phase shift $\delta$ can be related to the the neutron momentum $k$ via the effective-range expansion, which is given for $s$-wave interactions by

$$
k \cot \left(\delta_{S}\right)=-\frac{1}{a_{s}}+\frac{1}{2} r_{\text {eff }} k^{2}+\mathcal{O}\left(k^{4}\right),
$$

with the $s$-wave scattering length $a_{s}$ and the effective range $r_{\text {eff }}$.

Mathematically, the scattering length $a_{s}$ describes the slope of the phase shift at $k=0$. In particular, we use the common convention that negative signs of $a_{s}$ correspond to positive slopes and vice versa. Then a positive $s$-wave scattering length signals the existence of a bound state, c.f., the nuclear ${ }^{3} S_{1}$ partial wave, and a negative $a_{s}$ indicates no bound state, c. f., the ${ }^{1} S_{0}$ partial wave. Starting from a negative $a_{s}$, increasing the attraction of the interaction leads to a growing $\left|a_{s}\right|$. If $\left|a_{s}\right| \rightarrow \infty$, then a bound state appears at the threshold.

For neutron matter, $r_{\text {eff }} \approx 2.7 \mathrm{fm}$ and $a_{s}=-18.9 \mathrm{fm}$. Thus, the neutron-neutron interaction leads to no bound state in the $s$-wave channel but is nevertheless strongly attractive. At very low densities, $k_{\mathrm{F}} \ll 1$, the interparticle spacing is much larger than the effective range of the interaction. The system is then fully described by $k_{\mathrm{F}}$ and $a_{s}$. Systems with a similar $a_{s}$ and the same Fermi momentum $k_{\mathrm{F}}$ will experience the same physics. If in addition $a_{s}$ can be considered very large, or $\left(\left|a_{s} k_{\mathrm{F}}\right|\right)^{-1} \rightarrow 0$, then the system is completely described by the density or $k_{\mathrm{F}}$. This regime is called the universal regime, and the system's energy must be proportional to the energy of a free Fermi gas, hence

$$
E(n)=\xi_{0} E_{\mathrm{FG}}(n) .
$$

The UG, with $a_{s} \rightarrow \pm \infty$ and $r_{\text {eff }}=0$, has universal behavior but also will any dilute fermionic system with $r_{\text {eff }} \ll k_{\mathrm{F}}^{-1} \ll\left|a_{s}\right|$, e.g., neutron matter at low densities. In the unitary limit, the properties of the potential, e.g., its shape, become irrelevant and the system is completely described by the dimensionless combination $a_{s} k_{\mathrm{F}}$. $\xi_{0}$ can be measured in experiments with ultracold atoms (Ku et al. 2012; Zürn et al. 2013) around a Feshbach resonance. In this resonance, $a_{s}$ can be tuned over several orders of magnitude by varying the magnetic field.

For any fermionic system, unitary or not, we define $\xi=E / E_{\mathrm{FG}}$. Lee \& Yang (1957) showed that in the limit of zero density, $k_{\mathrm{F}} \rightarrow 0$ and $\left(k_{\mathrm{F}} a_{0}\right)^{-1} \ll-1$, which is far from the unitary limit:

$$
\xi=1+\frac{10}{9 \pi} k_{\mathrm{F}} a_{0}+\frac{(11-2 \ln 2)}{21 \pi^{2}}\left(k_{\mathrm{F}} a_{0}\right)^{2}+\cdots .
$$

Here, $a_{0}$ is the scattering length in the relevant channel. Near unitarity, $\left|k_{\mathrm{F}} a_{0}\right| \rightarrow \infty$, cold atom experiments (Navon et al. 2010) show that $\xi$ changes linearly with $\left(k_{\mathrm{F}} a_{0}\right)^{-1}$ for $\left|k_{\mathrm{F}} a_{0}\right| \gtrsim 50$; approximately

$$
\xi \simeq \xi_{0}-0.93\left(k_{\mathrm{F}} a_{0}\right)^{-1}+\cdots,
$$

which also compares favorably with theoretical predictions from QMC calculations of cold atoms (Carlson et al. 2012). Thus, forces with a finite negative $a_{s}$ at very low densities have $\xi>\xi_{0}$ and higher energies than the UG. Then for spin-one-half fermions interacting solely via the $s$-wave interactions that do not produce two-body bound states $\left(a_{s}<0\right)$, the unitary limit, $a_{s} \rightarrow-\infty$ and $r_{\mathrm{eff}} \rightarrow 0$, indicates the largest attraction. At very low densities, when neutron matter can be described solely by its $a_{s}$, the UG energy serves as a lower bound for $E_{\mathrm{PNM}}$ because the magnitude of the neutron $a_{s}$ is smaller, $\left|a_{s}\right|<\infty$, which indicates less attraction (Carlson et al. 2012; Gandolfi et al. 2015).

As stated above, at higher densities $s$-wave effective-range effects are not negligible anymore (e.g., $k_{\mathrm{F}} r_{\mathrm{eff}}=4.53$ at $n_{0}$ ). However, a finite effective range reduces the attraction and increases the energy per particle. For example, when $k_{\mathrm{F}} r_{\text {eff }} \lesssim 0.35$, the energy increases with density as $E=E_{\mathrm{FG}} \xi=E_{\mathrm{FG}}\left(\xi_{0}+\right.$ $\left.0.12(3) k_{\mathrm{F}} r_{\mathrm{eff}}+\ldots\right)$ (Gandolfi et al. 2015). At higher densities, up to at least $k_{\mathrm{F}} r_{\mathrm{eff}}=5$, correspondingly larger increases in $\xi$ have been found (Schwenk \& Pethick 2005). Because a finite $\left(a_{s} k_{\mathrm{F}}\right)^{-1}$ and a finite $r_{\text {eff }}$ both lead to an increase of the effective Bertsch parameter $\xi$, the energy of neutron matter is higher than for the UG, at least when only realistic $s$-wave interactions are considered.

At even higher densities, $p$ - and higher partial wave interactions may spoil this bound: the average $p$-wave interaction is very small but attractive, as are $d$-wave contributions to neutron matter.

When comparing the UG with neutron-matter calculations using only the full $\mathrm{NN}$ interactions, one finds that the energies of neutron matter for $n \lesssim n_{0}$ and the UG are comparable although the underlying interactions are very different. Softer (harder) NN interactions lead to slightly more (less) attraction compared to the UG. This may be due to fewer short-range correlations for softer interactions. In the $\mathrm{NN}$-only results of Tews et al. (2013), using soft chiral Hamiltonians, the neutronmatter energy at $n_{0}$ was found to be approximately $2 \mathrm{MeV}$ below that of the UG.

Because we found that some soft Hamiltonians violate the UG bound on the NN-only level, we performed additional MBPT calculations at third order similar to Tews et al. (2013), using two soft Hamiltonians and including only $s$-wave, $s+$ $p$-wave, and $s+p+d$-wave interactions. When considering only $s$-wave interactions we found that the energy per neutron lies $5-6 \mathrm{MeV}$ above the UG bound at $n_{0}$. This supports our justification for realistic $s$-wave interactions. For a finite $\ell$, we found that $p$ - and $d$-wave interactions contribute attractively and lower the energy to $2 \mathrm{MeV}$ below the UG at $n_{0}$. Higher partial waves $(\ell>2)$ add only a small contribution.

In neutron matter, however, several ab initio calculations have shown the importance of three-body $(3 \mathrm{~N})$ forces, which lead to, in net, additional repulsion, and increase the neutronmatter energy by several $\mathrm{MeV}$ at $n_{0}$. The inclusion of $3 \mathrm{~N}$ forces is sufficient to more than compensate for the $p$ - and $d$-wave attraction, so that the neutron-matter energy has values above the UG. In Figure 1 we show a comparison of the UG bound with several ab initio calculations for PNM, including both NN and 3N forces, of Drischler et al. (2016a), Lynn et al. (2016), Togashi \& Takano (2013), Gandolfi et al. (2012), Hebeler \& Schwenk (2010), Gezerlis \& Carlson (2010), Akmal et al. (1998), and Friedman \& Pandharipande (1981). Only one calculation violates the UG constraint within its uncertainty band: a QMC $\mathrm{N}^{2} \mathrm{LO}$ calculation using soft chiral forces (Lynn et al. 2016). This is due to artifacts from local regulators, which lead to less repulsion from $3 \mathrm{~N}$ forces (Dyhdalo et al. 2016; Tews et al. 2016), and is peculiar to that interaction.

We emphasize the fact that ab initio calculations with realistic $\mathrm{NN}$ and $3 \mathrm{~N}$ forces support our conjecture that 


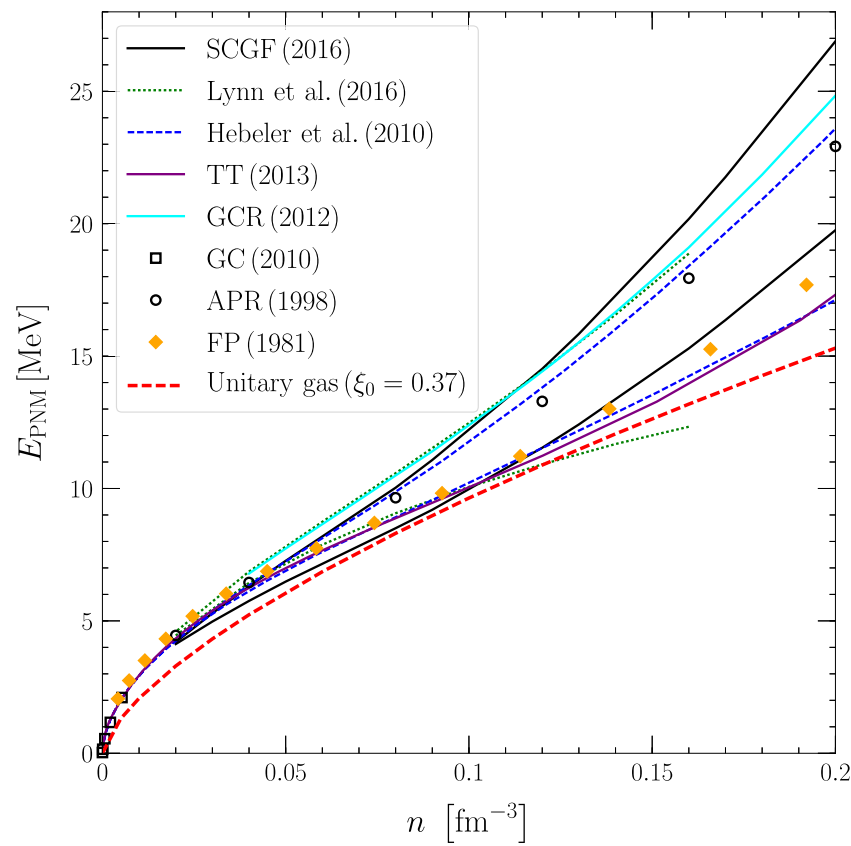

Figure 1. UG bound with $\xi_{0}=0.37$ compared to ab initio calculations of Drischler et al. (2016a) (SCGF), Lynn et al. (2016) (Lynn et al.), Hebeler \& Schwenk (2010) (Hebeler et al.), Togashi \& Takano (2013) (TT), Gandolfi et al. (2012) (GCR), Gezerlis \& Carlson (2010) (GC), Akmal et al. (1998) (APR), and Friedman \& Pandharipande (1981) (FP).

$E_{\mathrm{PNM}}>E_{\mathrm{UG}}$. Although the underlying interactions in both systems are rather different and thus it cannot be strictly proven that the UG is a lower bound for the neutron-matter energy at all densities, the combination of effective-range effects, small $p$-wave and tensor interactions, and repulsive $3 \mathrm{~N}$ forces in neutron matter strongly suggests that this conjecture is justified. It is the consequence of a realistic nuclear Hamiltonian and is not altered by the differences in interactions and wave functions within the two systems.

\section{The Minimal Constraint on the Symmetry Energy}

We now show what the inequality in Equation (12) implies for the symmetry energy parameters. At a baryon density of $n=u n_{0}$, the $\mathrm{UG}$ energy is

$$
E_{\mathrm{UG}}(u)=\frac{3}{5} E_{\mathrm{F}}(u) \xi_{0}=\frac{3 \hbar^{2} k_{\mathrm{F}}^{2}}{10 m_{n}} \xi_{0}=E_{\mathrm{UG}}^{0} u^{2 / 3},
$$

where $m_{n}$ is the neutron mass and $E_{\mathrm{UG}}^{0}=E_{\mathrm{UG}}(u=1) \simeq 12.6$ $\mathrm{MeV}$. The SNM energy $\left(E_{\mathrm{SNM}}\right)$ can be expanded as

$$
\begin{aligned}
E_{\mathrm{SNM}}(u)= & E_{0}+\frac{K_{0}}{18}(u-1)^{2}+\frac{Q_{0}}{162}(u-1)^{3} \\
& +\mathcal{O}\left[(u-1)^{4}\right],
\end{aligned}
$$

where $E_{0} \simeq-16 \mathrm{MeV}$ and $K_{0} \simeq 230 \mathrm{MeV}$ are the saturation energy and incompressibility parameters, respectively. The parameter $Q_{0} \sim-300 \mathrm{MeV}$ is the skewness parameter, whose value is not well known. Using Equation (1) for the definition of the symmetry energy, the conjecture (12) yields the lower

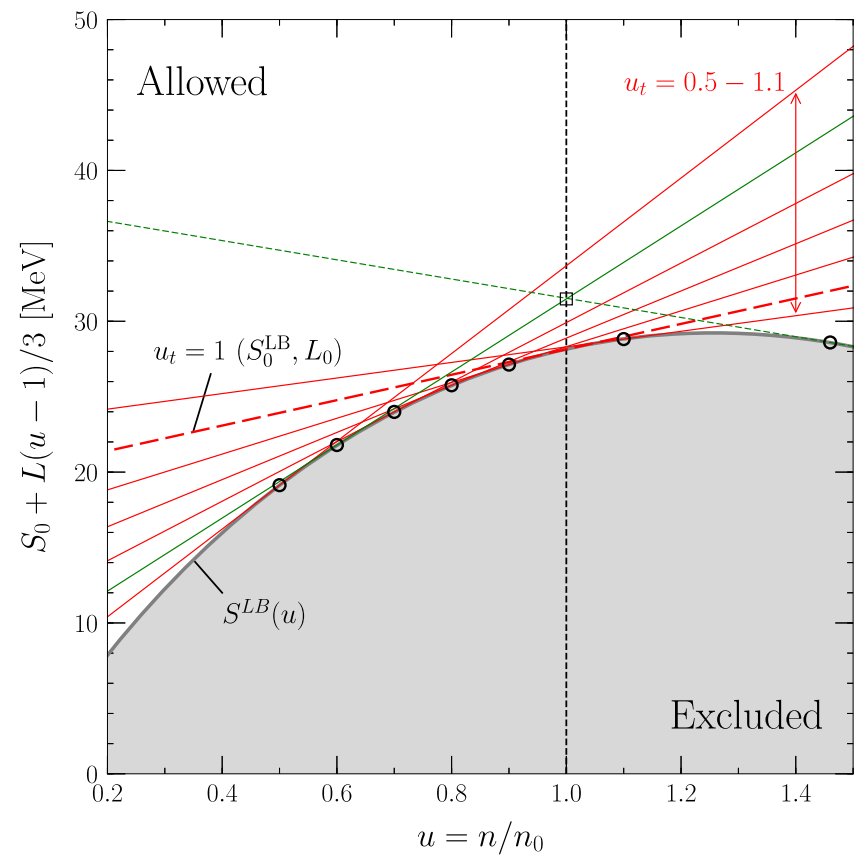

Figure 2. UG bound on the symmetry energy, using Equation (19) and typical parameters as described in the text. The shaded area is the region excluded for $S_{0}+L(u-1) / 3$. The dashed red curve shows the tangent curve to the boundary $S_{0}^{\mathrm{LB}}$ at $u_{t}=1$, for which $S_{0}=S_{0}^{\mathrm{LB}}$; other solid lines show the tangent curves for various values of $u_{t}$ (and $S_{0}$ ). The green curves show one particular case for $S_{0}>S_{0}^{\mathrm{LB}}$ : the solid green curve has $u_{t}=0.6$ and the dotted green curve has $u_{t}>1$.

bound $S^{\mathrm{LB}}(u)$ :

$$
\begin{aligned}
S(u) \geqslant & E_{\mathrm{UG}}^{0} u^{2 / 3}-\left[E_{0}+\frac{K_{0}}{18}(u-1)^{2}\right. \\
& \left.+\frac{Q_{0}}{162}(u-1)^{3}\right] \equiv S^{\mathrm{LB}}(u) .
\end{aligned}
$$

In Figure 2, we show the lower bound on the symmetry energy imposed by the UG constraint using typical values for the nuclear parameters $\left(E_{0}=-16 \mathrm{MeV}, K_{0}=230 \mathrm{MeV}\right.$, $Q_{0}=-300 \mathrm{MeV}$, and $\left.E_{\mathrm{UG}}^{0}=12.6 \mathrm{MeV}\right)$. The shaded area shows the excluded region, inside which the symmetry energy should not enter. The boundary of this region is quite insensitive to the values of $K_{0}$ and $Q_{0}$ : variations of $\Delta K_{0}= \pm 30 \mathrm{MeV}$ and $\Delta Q_{0}= \pm 300 \mathrm{MeV}$ each move the boundary at $u=0.2$ by only about $\pm 1 \mathrm{MeV}$, and less for values of $u$ closer to 1 .

From Equation (19), it is clear that $S_{0} \equiv S(u=1)$ is bounded from below: $S_{0} \geqslant E_{\mathrm{UG}}^{0}-E_{0} \equiv S_{0}^{\mathrm{LB}}$. Because the symmetry energy should not enter the excluded area, at $S_{0}=S_{0}^{\mathrm{LB}}$ the slopes of $S(u)$ and $S^{\mathrm{LB}}(u)$ must agree, as shown by the tangential red-dashed line in Figure 2. We thus find

$$
L=\left.3 u \frac{d S^{\mathrm{LB}}}{d u}\right|_{u=1}=2 E_{\mathrm{UG}}^{0} \equiv L_{0} .
$$

It also follows that the curvature of $S$ at $u=1$ must be greater than that of $S^{\mathrm{LB}}$ (which is negative), or else $S$ could penetrate 
into the excluded region for $u \neq 1$. This implies

$$
\begin{aligned}
K_{\mathrm{sym}} & \geqslant\left. 9 u^{2} \frac{d^{2} S^{\mathrm{LB}}}{d u^{2}}\right|_{u=1}=-2 E_{\mathrm{UG}}^{0}-K_{0} \equiv K_{\mathrm{sym}, 0}, \\
K_{n} & \geqslant-2 E_{\mathrm{UG}}^{0} \equiv K_{n, 0},
\end{aligned}
$$

where $K_{n}=K_{0}+K_{\text {sym }}$ is the incompressibility of PNM at saturation density. We also define the skewness of PNM at $u=1, Q_{n}=Q_{0}+Q_{\text {sym }}$. We find that $S_{0}^{\mathrm{LB}}, L_{0}$, and $K_{n, 0}$ are independent of $K_{0}$ and $Q_{0}$, the most uncertain of the saturation parameters, and thus appear to be very general.

By choosing the UG as a lower bound for neutron matter, we also pick a specific density dependence. We stress that a more realistic density dependence for the lower bound would be steeper than $u^{2 / 3}$ and lead to a less concave symmetry energy. ${ }^{7}$ In turn, this would lead to even more stringent constraints. Thus by choosing the particular density dependence $u^{2 / 3}$, we are suggesting a more conservative bound and are underestimating our constraint.

Using the expansion for $S(u)$ of Equation (11), allows Equation (19) to be expressed as

$$
\begin{aligned}
S_{0} & +\frac{L}{3}(u-1) \geqslant E_{\mathrm{UG}}^{0} u^{2 / 3}-\left[E_{0}+\frac{K_{n}}{18}(u-1)^{2}\right. \\
& \left.+\frac{Q_{n}}{162}(u-1)^{3}\right] .
\end{aligned}
$$

If we choose a value of $S_{0}$ that is greater than $S_{0}^{\mathrm{LB}}$, there exist two curves for $S(u)$ that can become tangent to $S^{\mathrm{LB}}(u)$ : one at a density of $u_{t}>1$ and the other at a density of $u_{t}<1$. Each curve thereby satisfies the inequality in Equation (22). For every value of $S_{0}>S_{0}^{\mathrm{LB}}$, there are thus two limiting values for $L$ that are each proportional to the slope of these tangential symmetry energy curves at $u=1$. The upper (or lower) bound for $L$ has a tangency density of $u_{t}<1$ (or $u_{t}>1$ ), as shown by the green lines in Figure 2. ${ }^{8}$ The conditions

$$
S\left(u_{t}\right)=S^{\mathrm{LB}}\left(u_{t}\right) \quad \text { and }\left.\quad \frac{d S}{d u}\right|_{u_{t}}=\left.\frac{d S^{\mathrm{LB}}}{d u}\right|_{u_{t}}
$$

at the tangency densities $u_{t}$ lead to the parametric equations

$$
\begin{aligned}
S_{0} & =\frac{E_{\mathrm{UG}}^{0}}{3 u_{t}^{1 / 3}}\left(u_{t}+2\right)+\frac{K_{n}}{18}\left(u_{t}-1\right)^{2}+\frac{Q_{n}}{81}\left(u_{t}-1\right)^{3}-E_{0}, \\
L & =\frac{2 E_{\mathrm{UG}}^{0}}{u_{t}^{1 / 3}}-\frac{K_{n}}{3}\left(u_{t}-1\right)-\frac{Q_{n}}{18}\left(u_{t}-1\right)^{2} .
\end{aligned}
$$

When $u_{t}=1$, we recover the bounds $S_{0}=S_{0}^{\mathrm{LB}}$ and $L=L_{0}$. For every value of $u_{t} \neq 1$, one can then determine a point of the boundary of the excluded region for $S_{0}(L)$, as shown in Figure $3 .^{9}$

The $\left(S_{0}, L\right)$ boundary depends on the saturation and UG parameters $n_{0}, E_{0}, K_{n}, Q_{n}$, and $\xi_{0}$ and becomes less

\footnotetext{
7 Equation (16) shows that $d \xi / d u>0$.

8 Note that for this example the lower bound for $L$ is negative.

9 By expanding $E_{\mathrm{UG}}(u)$ around $u=1$ to the second order and dropping the skewness terms, one can obtain an approximate but analytic form of this boundary as $S_{0}=E_{\mathrm{UG}}^{0}-E_{0}+\left(L-2 E_{\mathrm{UG}}^{0}\right)^{2} /\left[2\left(2 E_{\mathrm{UG}}^{0}+K_{n}\right)\right]$.
}

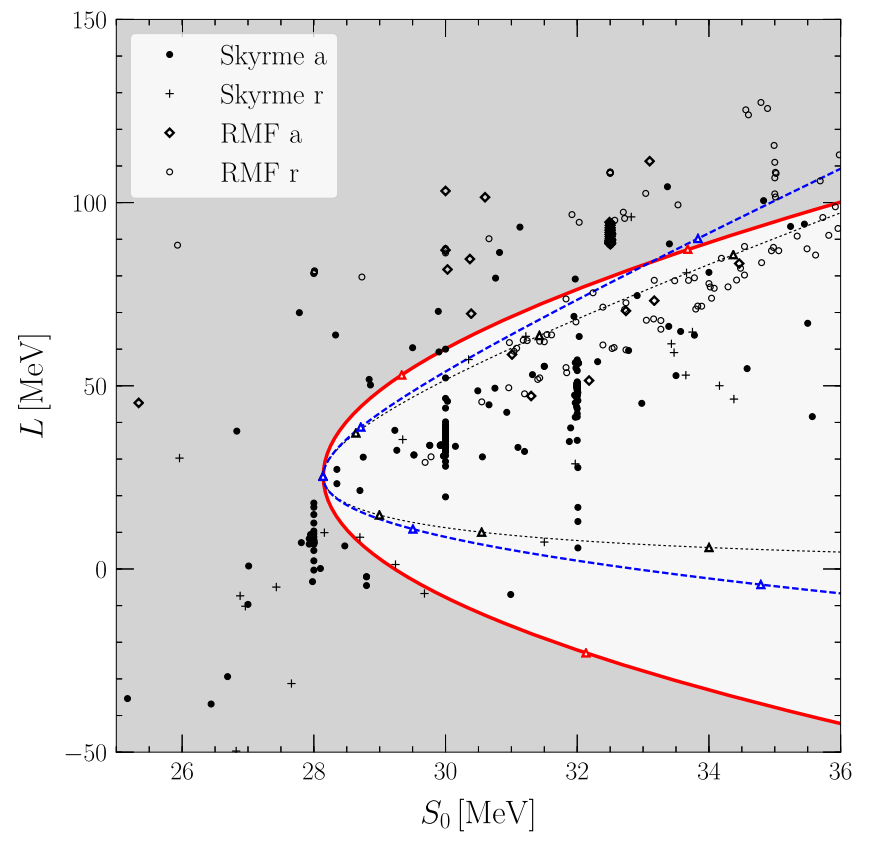

Figure 3. Exclusion boundaries from the conjecture $E_{\mathrm{PNM}}>E_{\mathrm{UG}}$. The solid red curve shows the fiducial bound from Equation (24) with the parameters of Equation (25); the shaded region is excluded. The dotted curve shows the analytic bound from Equation (32), and the dashed curve shows the bound as modified by Equations (33) and (34). Triangles along the curves, from top to bottom, show the points where $u_{t}=[0.4,0.5,0.75,1.0,1.5,2.0,3.0]$ (along the solid and dashed curves the points begin with $u_{t}=0.5$, and along the solid (dashed) curve no points with $u_{t}>1.5$ (2.0) are displayed). For reference, symmetry parameters from the compilations of Dutra et al. (2012, 2014) are indicated. The plus and open circle symbols (denoted "r") show interactions that were rejected, and the filled circles and open diamonds (denoted "a") show interactions that were accepted, as discussed later in Section 4 .

exclusionary the smaller $E_{\mathrm{UG}}^{0} \propto n_{0}^{2 / 3} \xi_{0}$, or the larger $E_{0}$ and $K_{n}$, or the smaller (larger) $Q_{n}$ for $u_{t}<1\left(u_{t}>1\right)$.

The Bertsch parameter for spin-half fermions is experimentally measured to be $\xi_{0}=0.376 \pm 0.004$ ( $\mathrm{Ku}$ et al. 2012) or $\xi_{0}=$ $0.370 \pm 0.005 \pm 0.008$ (Zürn et al. 2013). The values of the saturation parameters are $E_{0}=-15.9 \pm 0.4 \mathrm{MeV}, \quad n_{0}=$ $0.164 \pm 0.007 \mathrm{fm}^{-3}$ (Drischler et al. 2016b), and $K_{0}=240 \pm$ $20 \mathrm{MeV}$ (Shlomo et al. 2006; Piekarewicz 2010) or $K_{0}=230 \pm$ $40 \mathrm{MeV}$ (Khan et al. 2012). In general, $K_{\mathrm{sym}}<0$ for realistic relativistic mean-field (RMF) and Skyrme forces, i.e., those that have been fit to properties of laboratory nuclei. Realistic microscopic interactions also suggest $K_{\text {sym }}<0$ : e.g., $\mathrm{N}^{3} \mathrm{LO}$ chiral EFT calculations (Tews et al. 2013) yield $K_{n}=119 \pm 101 \mathrm{MeV}$ (Margueron et al. 2017). The neutron matter calculations of Drischler et al. (2016b) yield $-70 \mathrm{MeV}>K_{\text {sym }}>-240 \mathrm{MeV}$ and $10 \mathrm{MeV}<K_{n}<100 \mathrm{MeV}$. Since both experimental data and theoretical neutron matter calculations indicate that $K_{\text {sym }}<0$, it follows that $K_{0}>K_{n}>K_{n, 0}$.

Experimental constraints on $Q_{0}$ are weak. Cai \& Chen (2014) have argued, based on heavy-ion flow data analyzed by Danielewicz et al. (2002) and the existence of $2 M_{\odot}$ neutron stars, that $-494 \mathrm{MeV}<Q_{0}<-10 \mathrm{MeV}$. This range is consistent with that suggested by the neutron matter calculations of Drischler et al. (2016b), $-450 \mathrm{MeV}<Q_{0}<-50 \mathrm{MeV}$. Fitting the energies of the giant monopole resonance, Farine et al. (1997) argue that $-1200 \mathrm{MeV}<Q_{0}<-200 \mathrm{MeV}$, which is also consistent with these other results. Constraints on $Q_{\text {sym }}$ and $Q_{n}$ are even weaker than for $K_{n}$, but the neutron matter 

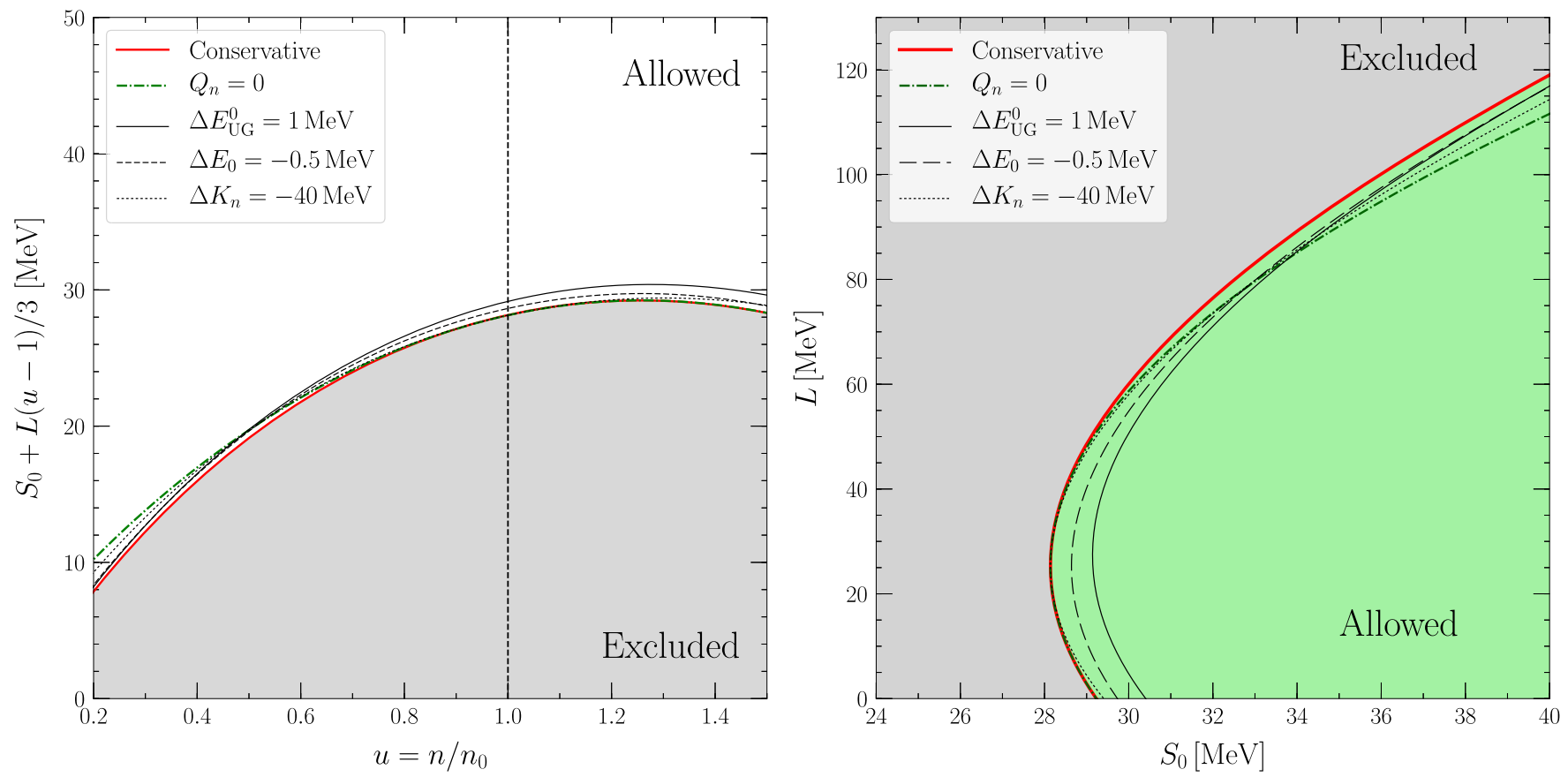

Figure 4. Left panel: The parameter dependence of the bound for the symmetry energy. Right panel: The parameter dependence of the bounds for the symmetry energy parameters $S_{0}$ and $L$. In each panel, the solid curve shows the bound with the conservative parameter set, per Equation (25). Dotted-dashed, thin solid, dashed, and dotted lines show the bounds with $\Delta Q_{n}=750 \mathrm{MeV}$ (for $u_{t}<1$ ), $\Delta E_{\mathrm{UG}}^{0}=1 \mathrm{MeV}, \Delta E_{0}=-0.5 \mathrm{MeV}$, and $\Delta K_{n}=-40 \mathrm{MeV}$, respectively.

calculations of Drischler et al. (2016b) give $-750 \mathrm{MeV}<Q_{n}<$ $-250 \mathrm{MeV}$.

From these parameter ranges and Equation (22), $K_{n}=K_{0}$ is a conservative (least exclusionary) choice for the bound. Since $\left(u_{t}-1\right)$ changes sign at $u_{t}=1, Q_{n}=0$ is a conservative choice for $u_{t}>1$ while $Q_{n}=-750 \mathrm{MeV}$ is a conservative choice for $u_{t}<1$. Thus we adopt

$$
\begin{aligned}
E_{0} & =-15.5 \mathrm{MeV}, n_{0}=0.157 \mathrm{fm}^{-3}, K_{n}=K_{0}=270 \mathrm{MeV}, \\
K_{\text {sym }} & =0, Q_{n}=0 \mathrm{MeV} \text { or }-750 \mathrm{MeV}, \xi_{0}=0.365
\end{aligned}
$$

as the fiducial conservative parameter set. This results in $E_{\mathrm{UG}}^{0}=12.64 \mathrm{MeV}, S_{0}^{\mathrm{LB}}=28.14 \mathrm{MeV}, L_{0}=25.28 \mathrm{MeV}$, and $K_{n, 0}=-25.28 \mathrm{MeV}$.

Figure 3 shows the bounds on $S_{0}$ and $L$ from the UG constraint with the fiducial parameter set. Points for several values of $u_{t}$ are shown, e.g., the point where $u_{t}=1 / 2$ is indicated, for which $L \simeq 87 \mathrm{MeV}$ and $S_{0} \simeq 33.6 \mathrm{MeV}$. This value of $u_{t}$ could represent a plausible limit of applicability of the Taylor expansion of Equation (11). ${ }^{10}$ From Equation (24) one finds that $L=0 \mathrm{MeV}$ when $u_{t}=1.26$. Therefore, it appears that our bounds should be reliable for most of the figure since $u_{t}$ remains relatively close to unity throughout.

Figure 4 shows the effect of changing assumptions about the saturation and unitarity parameters. Note that only variations in $E_{0}, K_{n}, Q_{n}$, and $E_{\mathrm{UG}}^{0}$ need to be considered. Results are shown for the variations $\Delta E_{\mathrm{UG}}^{0}=+1 \mathrm{MeV}, \Delta E_{0}=-0.5 \mathrm{MeV}$, and $\Delta K_{n}=-40 \mathrm{MeV}$, as well as for $\Delta Q_{n}=+750 \mathrm{MeV}$ when $u_{t}<1$. Variations caused by $\left|\Delta Q_{n}\right| \sim 300 \mathrm{MeV}$ are similar to those from changing $K_{n}$ by about $30 \mathrm{MeV}$. Varying $E_{0}$ or $E_{\mathrm{UG}}^{0}$ translates directly into a movement of the point $\left(S_{0}^{\mathrm{UB}}, L_{0}\right)$ and

\footnotetext{
$\overline{{ }^{10} \text { Equation (11) predicts } S(u \rightarrow 0)=S_{0}}-L / 3+K_{\text {sym }} / 18-Q_{\text {sym }} / 162$, whereas $S(u \rightarrow 0)$ should in fact vanish.
}

thus produces the largest variations in the exclusion boundary near $u_{t} \sim 1$. In this region, variations within the large uncertainties for $K_{n}$ and $Q_{n}$ are negligible. These uncertainties only become significant when $u_{t}$ is appreciably different from unity, where the validity of our bounds is not assured. Nevertheless, variations of $K_{n}$ of the order of $30 \mathrm{MeV}$ and $Q_{n}$ of the order of $300 \mathrm{MeV}$ translate into boundary shifts of no more than $\sim 1 \mathrm{MeV}$ in $S_{0}$ for a given value of $L$, or $\sim 3 \mathrm{MeV}$ in $L$ for a given value of $S_{0}$, even for the extreme case $\left|u_{t}-1\right| \simeq 0.8$. We conclude that the fiducial boundary is remarkably insensitive to uncertainties in the saturation parameters and the Bertsch parameter.

Zhang et al. (2017) recently argued that the symmetry energy bounds are much more uncertain than we have suggested because the uncertainties in $\xi_{0}, K_{0}, K_{\text {sym }}, Q_{0}$, and $Q_{\text {sym }}$ are much greater than proposed here. For example, Zhang et al. (2017) argued that the uncertainty in $\xi_{0}$ is about 0.1 , but this is not supported by recent cold atom experiments, as discussed in this section. Furthermore, we have found that the individual parameters $K_{0}, K_{\mathrm{sym}}, Q_{0}$, and $Q_{\text {sym }}$ themselves are not particularly relevant for the symmetry bounds. Rather, it is the combinations $K_{n}=K_{0}+K_{\text {sym }}$ and $Q_{n}=Q_{0}+Q_{\text {sym }}$ that directly appear. Moreover, the bulk of nuclear interactions developed over decades to fit nuclear properties show pronounced correlations among $K_{n}, Q_{n}$, and $L$. As a result, the large individual parameter uncertainties do not play as important a role as may have been thought. A more realistic symmetry bound incorporating these correlations is addressed in the next section.

\section{More Realistic Constraints on the Symmetry Energy Parameters}

The previous section described a minimal constraint for the symmetry energy parameters as obtained from relatively conservative choices for the saturation properties of matter 


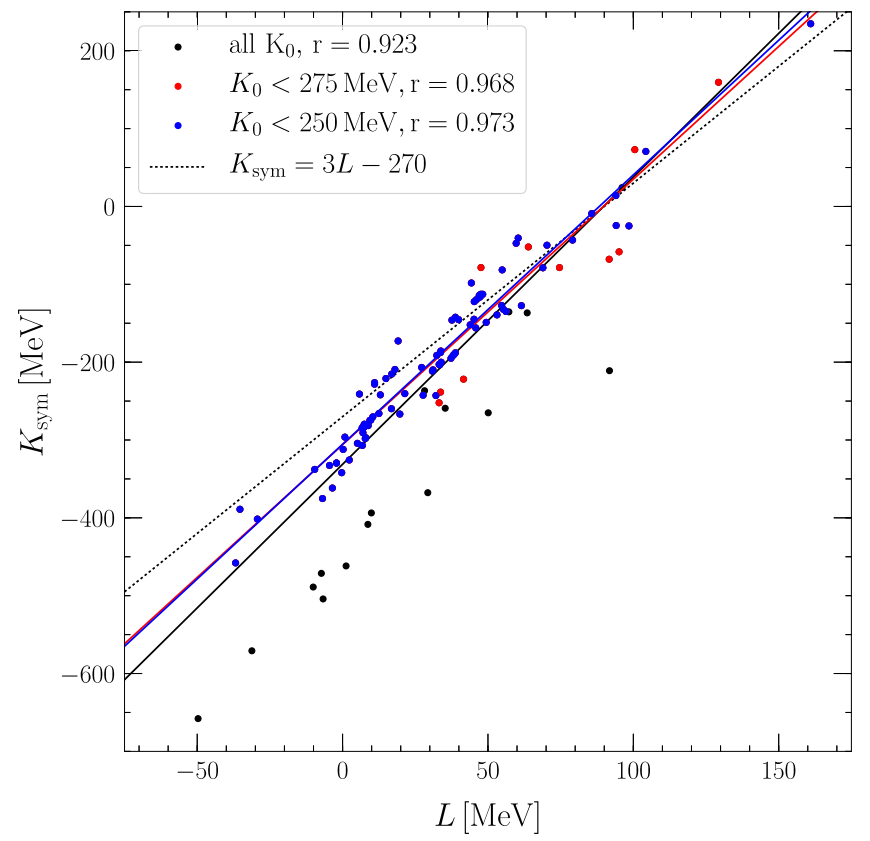

Figure 5. Correlations between $K_{\text {sym }}$ and $L$ for 118 Skyrme forces studied by Danielewicz \& Lee (2009). Colors distinguish forces according to the incompressibility parameters $K_{0}$. Their respective correlation coefficients are given by $r$.

and the Bertsch parameter. The largest parameter uncertainties exist for $K_{\text {sym }}, Q_{0}$, and $Q_{\text {sym }}$. In this section, we discuss the evidence that these parameters are correlated with each other and with $L$. Such collective correlations can reduce the variations introduced by the uncertainties of the individual parameters. The use of these correlations results in a more phenomenological but possibly more realistic symmetry energy bound.

In our fiducial estimate, we conservatively chose $K_{\text {sym }}=0$. More realistically, however, $K_{\text {sym }}<0$. It has also been found that realistic forces of both nonrelativistic potential and relativistic field-theoretical types that are calibrated by fitting energies and charge radii of laboratory nuclei show a linear correlation between $K_{\text {sym }} / S_{0}$ and $L / S_{0}$ (Danielewicz \& Lee 2009) or between $K_{\text {sym }}$ and $L$ (Chen et al. 2009; Vidaña et al. 2009; Ducoin et al. 2011). ${ }^{11}$ Specifically, Danielewicz \& Lee (2009) studied 118 Skyrme forces and found the correlations to be bracketed by the expressions

$$
K_{\text {sym }} / S_{0}=6 L / S_{0}-18 \quad \text { and } \quad K_{\text {sym }} / S_{0}=4 L / S_{0}-10,
$$

while Chen et al. (2009) studied 63 Skyrme interactions and the MDI force and found the bracketing expressions

$$
K_{\text {sym }}=5 L-500 \mathrm{MeV} \quad \text { and } \quad K_{\text {sym }}=4 L-250 \mathrm{MeV} \text {. }
$$

However, studying the same interactions as Danielewicz \& Lee (2009), we have found the correlation

$$
K_{\text {sym }} \simeq 3.69 L-331.2 \pm 41.5 \mathrm{MeV}, \quad r=0.923 .
$$

The poor quality of this correlation is largely due to the wide range of $K_{0}$ values among the forces. As observed in Figure 5,

\footnotetext{
11 Since the variation of $S_{0}$ among different forces is relatively small, these correlations are essentially the same.
}

when interactions with $K_{0}>275$ (250) $\mathrm{MeV}$ are excluded from consideration, the correlation is considerably tightened. We have found

$$
\begin{aligned}
& K_{\text {sym }} \simeq 3.41 L-306.0 \pm 28.3 \mathrm{MeV} \\
& r=0.968\left(K_{0}<275 \mathrm{MeV}\right) \\
& K_{\text {sym }} \simeq 3.46 L-305.5 \pm 26.4 \mathrm{MeV}, \\
& r=0.973\left(K_{0}<250 \mathrm{MeV}\right),
\end{aligned}
$$

where the uncertainty is such that $68.3 \%$ of the interactions with $K_{0} \leqslant 275$ (250) MeV lie within these bounds. We verified that similar correlations also exist between $K_{\text {sym }} / S_{0}$ and $L / S_{0}$, which are slightly more significant.

The facts that the intercept of this correlation is close to our upper limit to $K_{0}$ and that the slope is close to 3 fortuitously allow the simplification $K_{n} \simeq 3 L$. With this expression, the boundary in Equation (24) can be analytically expressed (still assuming $Q_{n}=0$ ) as

$$
S_{0}=\frac{E_{\mathrm{UG}}^{0}}{3} \frac{1+2 u_{t}^{2}}{u_{t}^{4 / 3}}-E_{0}, \quad L=\frac{2 E_{\mathrm{UG}}^{0}}{u_{t}^{4 / 3}} .
$$

Eliminating $u_{t}$, one finds, simply,

$$
S_{0}=\frac{L}{6}\left[1+2\left(\frac{2 E_{\mathrm{UG}}^{0}}{L}\right)^{3 / 2}\right]-E_{0} .
$$

This analytic exclusion boundary is compared to the fiducial conservative boundary in Figure 3 . We note that the analytic exclusion boundary does not allow negative values of $L$.

We now examine correlations among the parameters in more detail. A collection of 240 Skyrme forces was studied by Dutra et al. (2012) and another collection of $263 \mathrm{RMF}$ forces by Dutra et al. (2014). Using these compilations, we have found that $K_{n}, Q_{n}$, and $L$ are strongly correlated. However, these compilations, especially the one containing RMF interactions, include many parametrizations with very unrealistic saturation properties. Keeping the 188 Skyrme and 73 RMF interactions with $0.149 \mathrm{fm}^{-3}<\rho_{0}<0.17 \mathrm{fm}^{-3}$, $-17 \mathrm{MeV}<E_{0}<-15 \mathrm{MeV}, 25 \mathrm{MeV}<S_{0}<36 \mathrm{MeV}$, and $180 \mathrm{MeV}<K_{0}<275 \mathrm{MeV}$ (accepted interactions) and rejecting the others, we find the following correlations with the respective correlation coefficients $r$ :

$$
\begin{aligned}
& K_{n}=3.534 L-(74.02 \pm 21.17[61.84]) \mathrm{MeV}, \\
& r=0.96,
\end{aligned}
$$

and

$$
\begin{aligned}
& Q_{n}=-7.313 L+(354.03 \pm 133.16[216.3]) \mathrm{MeV}, \\
& r=-0.78
\end{aligned}
$$

The quoted errors correspond to displacements in a correlation line that will enclose $68.3 \%$ of the interactions $(95.4 \%$ for the error displayed in square brackets). We show correlations in Figure 6. In this figure, as well as in Figures 3, 7 and 8, many of the "rejected" interactions fall outside the boundaries of the plot.

Note that $K_{n}$ and $L$ are especially highly correlated $(r=0.96)$. Furthermore, these results indicate that $K_{n}$ and $Q_{n}$ are negatively correlated while their errors are positively correlated: an interaction that has a $K_{n}$ value greater than the mean for its $L$ value also tends to have a $Q_{n}$ value larger than 

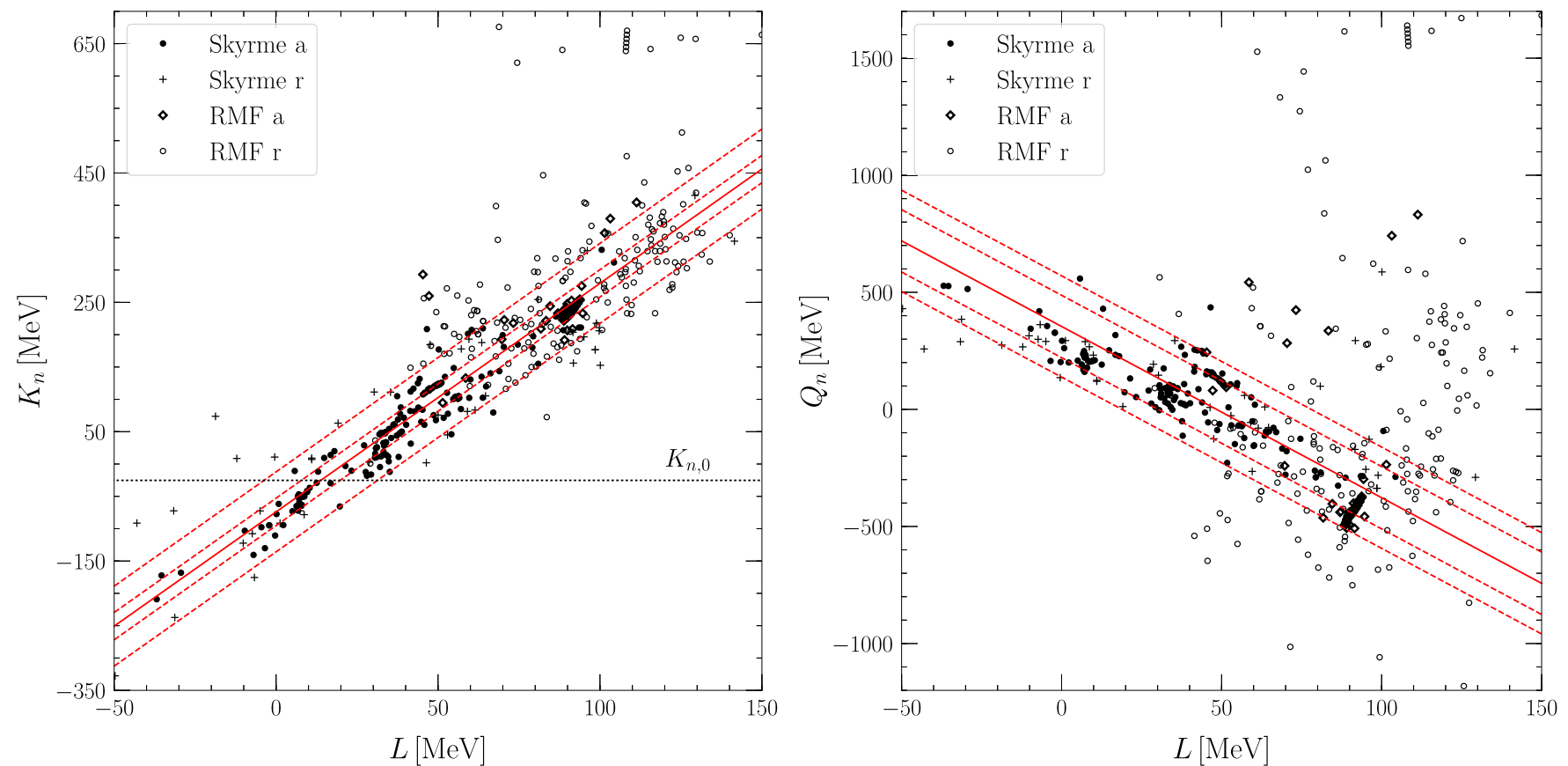

Figure 6. The left (right) panel shows the neutron-matter incompressibility $K_{n}$ (skewness $Q_{n}$ ) vs. the symmetry parameter $L$ for 240 Skyrme interactions compiled by Dutra et al. (2012) and 263 RMF forces compiled by Dutra et al. (2014). The solid lines show the correlations obtained from the "accepted" (a) realistic interactions after excluding the "rejected" (r) interactions; the dashed lines enclose, respectively, $68.3 \%$ and $95.4 \%$ of the accepted interactions. The dotted line in the left panel shows the minimum value $-25.28 \mathrm{MeV}$ for $K_{n}$ permitted by the conjecture that $E_{\mathrm{PNM}} \geqslant E_{\mathrm{UG}}$.

the mean. In the left panel of Figure 6, we also indicate the minimum value of $K_{n}$ permitted by the conjecture that $E_{\mathrm{PNM}} \geqslant E_{\mathrm{UG}}$, namely $K_{n, 0}=-25.28 \mathrm{MeV}$. Using the correlation of Equation (33), this implies a lower limit to $L$, $-3.7 \mathrm{MeV}$.

In a similar way, the parameters $S_{0}, K_{\mathrm{sym}}, Q_{\mathrm{sym}}$, and $L$ are also strongly correlated:

$$
L=11.969 S_{0}-(319.55 \pm 19.83[41.56]) \mathrm{MeV}, r=0.74
$$

$$
\begin{aligned}
& K_{\text {sym }}=3.501 L-(305.67 \pm 24.26[56.59]) \mathrm{MeV}, \\
& r=0.96,
\end{aligned}
$$

and

$$
\begin{aligned}
& Q_{\text {sym }}=-6.443 L+(708.74 \pm 118.14[171.34]) \mathrm{MeV}, \\
& r=-0.86,
\end{aligned}
$$

as depicted in Figures 7 and 8. Note that the correlation between $K_{\text {sym }}$ and $L$ is not surprisingly nearly the same as that found from the compilation of Danielewicz \& Lee (2009), Equation (30). As for $K_{n}$ and $Q_{n}$, there is a negative correlation between $K_{\text {sym }}$ and $Q_{\text {sym }}$ as well as a positive correlation in their errors.

Note that correlations among the parameters are not as strong for RMF approaches as they are for the nonrelativistic Skyrme models. It is also noteworthy that a large fraction of these Skyrme interactions $(22 \%)$ but an even larger fraction of the RMF forces $(73 \%)$ have unrealistic saturation properties as established from fitting nuclear masses, e.g., using liquiddroplet mass formula fits (and we have, hence, "rejected" them). Therefore, such compilations should be used with caution. Zhang et al. (2017) used the same compilations to argue that uncertainties in $K_{n}$ and $Q_{n}$ are too large to definitely constrain the parameters $S_{0}$ and $L$ from UG arguments, but they did not reject unrealistic interactions. Curiously, the correlation between $K_{n}$ and $Q_{n}$ is apparent in their study even without any rejections, although it was not utilized.

Finally, a more realistic exclusion boundary is obtained by substituting Equations (33) and (34), both with their upper 95.4\% error bars, into Equation (24). This allows the elimination of $K_{n}$ and $Q_{n}$ so that once again $S_{0}$ and $L$ are solely related through the parametric variable $u_{t}$. This modified boundary is also shown in Figure 3 in comparison to the fiducial boundary from Equation (24) and the analytic boundary given by Equation (32). The modified boundary closely follows the analytic boundary. We emphasize that the errors of the correlations between $K_{n}$ and $L$ and between $Q_{n}$ and $L$ are small enough that these boundaries are affected at the $1 \mathrm{MeV}$ level only for $\left|u_{t}-1\right| \gtrsim 0.2$, at which point the Taylor expansion of the energies is probably unreliable. Thus the analytic boundary in Equation (32) is a good approximation for the realistic boundary provided by UG constraints plus correlations among $K_{n}, Q_{n}$, and $L$.

\section{Comparison with Experimental Constraints}

The most abundant, accurate, and model-free experimental constraint on symmetry energy parameters comes from nuclear binding energies. As discussed by Lattimer \& Lim (2013), the resulting correlation between $S_{0}$ and $L$ is nearly linear. A recent Hartree-Fock study by the UNEDF collaboration (Kortelainen et al. 2010) found the confidence ellipse shown in Figure 8, assuming a fiducial fitting error of $2 \mathrm{MeV}$ for the nuclear binding energies. A nearly identical result was found from a liquid-droplet analysis (Lattimer \& Lim 2013). This confidence ellipse is essentially compatible with the UG constraint (for positive values of $L$ ). Even though a large fraction of the parameter sets taken from the compilations of Dutra et al. 

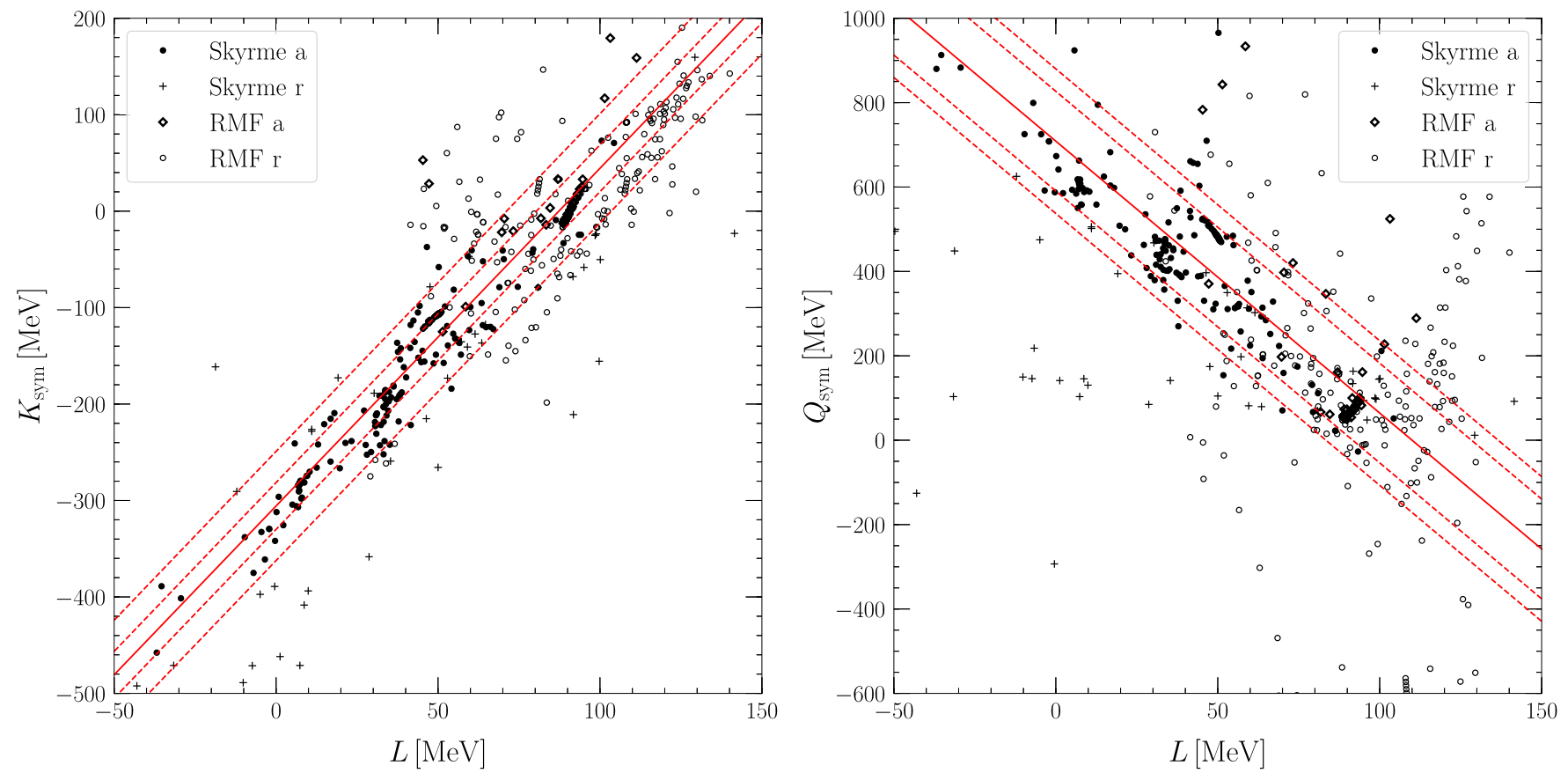

Figure 7. The left (right) panel shows the symmetry incompressibility $K_{\text {sym }}$ (skewness $Q_{\text {sym }}$ ) vs. the symmetry parameter $L$ for 240 Skyrme interactions compiled by Dutra et al. (2012) and 263 RMF forces compiled by Dutra et al. (2014). The solid lines show the correlations obtained from the "accepted" (a) realistic interactions after excluding the "rejected" ( $r$ ) interactions; the dashed lines enclose, respectively, $68.3 \%$ and $95.4 \%$ of the accepted interactions.

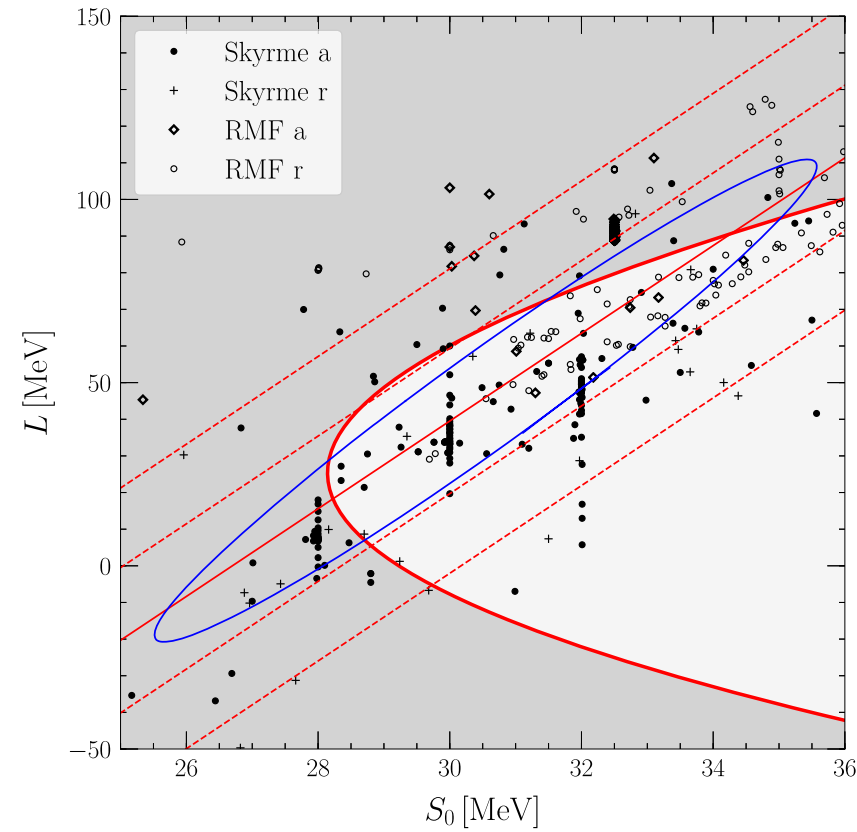

Figure 8. The confidence ellipse for the $S_{0}-L$ correlation as determined by Kortelainen et al. (2010) for an assumed fiducial binding energy error of $2 \mathrm{MeV}$. The solid red curve shows the conservative fiducial exclusion boundary from Equation (24) with the parameter set from Equation (25). For reference, the symmetry parameters from the compilations of Dutra et al $(2012,2014)$ are indicated, as in Figure 6. The correlation between these parameters for the "accepted" interactions of Equation (35) is shown as the solid line, together with the dashed lines showing $68.3 \%$ and $95.4 \%$ enclosures.

(2012, 2014) do not satisfy the UG constraint, a larger fraction of the accepted parameter sets do obey this constraint; furthermore, they show almost exactly the same correlation as in the analysis of Kortelainen et al. (2010). Combined, the
UG and nuclear binding-energy constraints imply that $L<100 \mathrm{MeV}$ and $S_{0}<35 \mathrm{MeV}$.

Other experimental constraints on symmetry energy parameters are reviewed in Lattimer \& Lim (2013) and Oertel et al. (2017). They indicate that consistency with measurements of nuclear masses, giant dipole resonances and dipole polarizabilities, neutron-skin thicknesses, and flows in heavy-ion collisions is achieved for $30 \mathrm{MeV} \leqslant S_{0} \leqslant 32 \mathrm{MeV}$ and $40 \mathrm{MeV} \leqslant L \leqslant 60 \mathrm{MeV}$ (left panel of Figure 9). ${ }^{12}$ It is observed that these ranges for $S_{0}$ and $L$ are compatible with neutron-matter calculations and both conservative and realistic UG bounds. Recently, Danielewicz et al. (2017) have argued, using isobaric analog states and isovector skins on neutron-rich nuclei, that both symmetry parameters may be larger than this consensus. Their $68 \%$ confidence region, depicted in the left panel of Figure 9, does not coincide with the overlap region from Lattimer \& Lim (2013) but is still mostly compatible with our UG constraint. This figure emphasizes the importance of both theoretical neutron-matter calculations, suitably calibrated by the energies of light nuclei, and measurements of neutronskin thicknesses in establishing an upper limit to the parameter $L$.

\section{Applications}

In the following we demonstrate the significance of the UG bound for astrophysics. In the right panel of Figure 9 we plot the values of the symmetry energy parameters for 10 tabulated equations of state (Fischer et al. 2014) frequently used for astrophysical simulations. Note that half of them violate the bound, which highlights the need for additional equation of

\footnotetext{
${ }^{12}$ In the left panel of Figure 9, the results of Kortelainen et al. (2010) are displayed using a fiducial fitting error of $1 \mathrm{MeV}$, as using $2 \mathrm{MeV}$ leads to negative $L$ values for small $S_{0}$.
} 

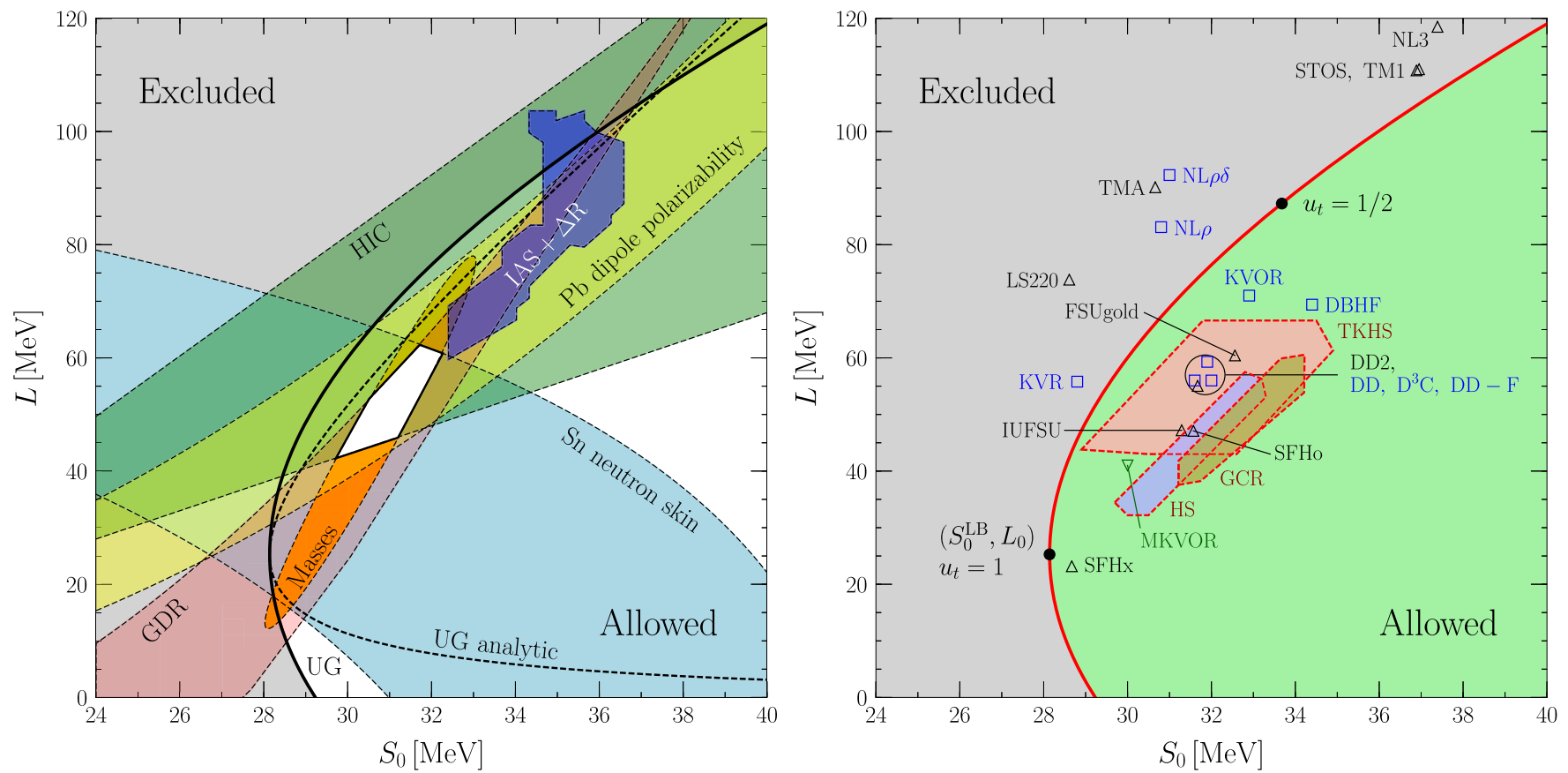

Figure 9. UG bounds on symmetry energy parameters. The thick lines show the bound Equation (24) using the conservative parameter set of Equation (25). Excluded regions are shown by shading. Left panel: Experimental constraints are from Lattimer \& Lim (2013) and Lattimer \& Steiner (2014), supplemented by isobaric analog states and isovector skin (IAS $+\Delta R$ ) results from Danielewicz et al. (2017). The thick dashed curve shows the analytic bound from Equation (32). Right panel: Filled circles show the point $\left(S_{0}^{\mathrm{LB}}, L_{0}\right)$ at the tangent density $u_{t}=1$ and the point where $u_{t}=1 / 2$. Triangles show values for interactions commonly used in tabulated equations of state for astrophysical simulations (notation and data from Fischer et al. 2014), and open squares (from Klähn et al. 2006) and the inverted triangle (from Maslov et al. 2016) show those of other frequently used interactions. The shaded regions TKHS, GCR, and HS show the parameter ranges inferred from the PNM calculations of Tews et al. (2013), Gandolfi et al. (2012), and Hebeler et al. (2010), respectively.

state tables that satisfy these conservative constraints. We also display the results for other interactions (Klähn et al. 2006; Maslov et al. 2016) commonly used in astrophysics and heavyion physics, among which a nontrivial number are found to violate the bound. We emphasize that realistic uncertainties in the relevant parameters $\xi_{0}, n_{0}, E_{0}, K_{n}$, and $Q_{n}$ do not affect these conclusions in any significant fashion.

Furthermore, the lower limit on the symmetry energy, implied by the UG constraint for $u<1$, has implications for the surface energy of nuclei, the location of the crust-core boundary, and the radii and moments of inertia of neutron stars. We show herein that this lower limit will establish maxima to the surface symmetry energy parameter $S_{S}$ and minima to neutron-star radii and moments of inertia. Curiously, although our conjecture $E_{\mathrm{PNM}}>E_{\mathrm{UG}}$ essentially determines a minimum for the symmetry energy, it also implies a maximum limiting behavior for $u \geqslant 1$. This has implications for the threshold density for the onset of rapid neutrino cooling due to the nucleon Urca process and, thus, for neutron-star cooling.

To investigate these applications of the UG bound, we require a better parameterization of $S$ than that given by the expansion of Equation (11), which fails in the limits of both small and large $u$. Instead, we model the symmetric matter and symmetry energy using these expressions:

$$
E_{\mathrm{SNM}}=T\left[u^{2 / 3}+a^{\prime} u+b^{\prime} u^{4 / 3}+c^{\prime} u^{5 / 3}+d^{\prime} u^{2}\right]
$$

and

$$
S(u)=T\left[\left(2^{2 / 3}-1\right) u^{2 / 3}+a u+b u^{4 / 3}+c u^{5 / 3}+d u^{2}\right] .
$$

The parameters are fit to properties of matter at saturation density $(u=1)$ :

$$
\begin{aligned}
& a^{\prime}=-4+\frac{120 E_{0}+6 K_{0}-Q_{0}}{6 T}, \\
& b^{\prime}=6+\frac{-90 E_{0}-5 K_{0}+Q_{0}}{2 T}, \\
& c^{\prime}=-4+\frac{72 E_{0}+4 K_{0}-Q_{0}}{2 T}, \\
& d^{\prime}=1+\frac{-60 E_{0}-3 K_{0}+Q_{0}}{6 T}, \\
& a=-4\left(2^{2 / 3}-1\right)+\frac{120 S_{0}-38 L+6 K_{\mathrm{sym}}-Q_{\mathrm{sym}}}{6 T}, \\
& b=6\left(2^{2 / 3}-1\right)+\frac{-90 S_{0}+30 L-5 K_{\mathrm{sym}}+Q_{\mathrm{sym}}}{2 T}, \\
& c=-4\left(2^{2 / 3}-1\right)+\frac{72 S_{0}-24 L+4 K_{\mathrm{sym}}-Q_{\mathrm{sym}}}{2 T}, \\
& d=2^{2 / 3}-1+\frac{-60 S_{0}+20 L-3 K_{\mathrm{sym}}+Q_{\mathrm{sym}}}{6 T} .
\end{aligned}
$$

Because the value of $Q_{0}$ is quite uncertain we use $d^{\prime}=0$, which implies $Q_{0}=-432.3 \mathrm{MeV}$ for the typical values $E_{0}=-16 \mathrm{MeV}, K_{0}=220 \mathrm{MeV}$, and $n_{0}=0.16 \mathrm{fm}^{-3}$. This value matches the means of the values of $Q_{n}-Q_{\text {sym }}$ for the interactions displayed in Figures 6 and 7 for $L \sim 50 \mathrm{MeV}$.

In this section, we always make use of this parametrization for the symmetry energy. While this parametrization allows the use of any reasonable value for the empirical parameters and thus does not automatically lead to any correlations between these parameters, this does not necessarily imply the absence of 


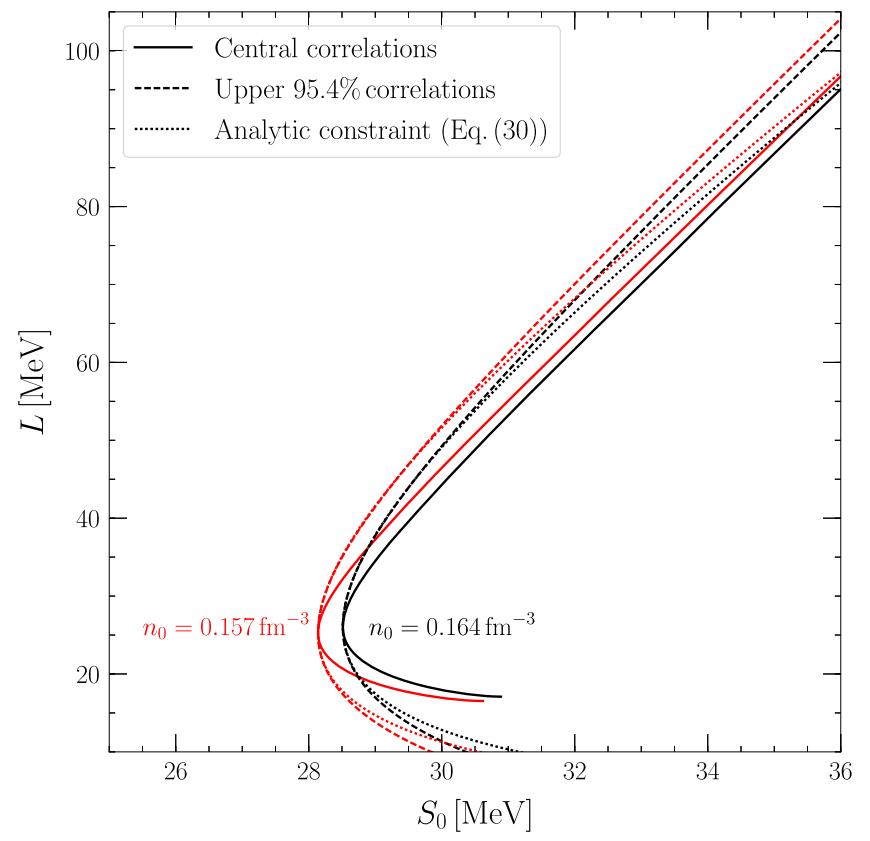

Figure 10. Constraints on the symmetry parameters from the power-law energy-density functionals of Equations (38) and (39) are shown by solid and dashed black (red) curves for $n_{0}=0.164(0.157) \mathrm{fm}^{-3}$ and $E_{0}=-15.5 \mathrm{MeV}$. The solid (dashed) curves correspond to the incorporation of the $K_{n}-L$ and $Q_{n}-L$ central (upper 95\%) correlations. The black (red) dotted curves show the corresponding analytic constraints from Equation (32).

physical correlations between the parameters. These correlations, as suggested by other realistic parametrizations investigated in Section 4, are a result of correctly describing nuclear systems. In other words, some parameter choices in Equation (40) will lead to unphysical behavior. Thus in the following, we utilize in addition the correlations between $K_{\text {sym }}$, $Q_{\text {sym }}$, and $L$. Then one can find the UG bound $L\left(S_{0}\right)$ using the tangency conditions of Equation (23). For a given value of $u_{t}$, this results in two equations linear in $S_{0}$ and $L$. The most conservative bound is found utilizing the upper $95.4 \%$ errors for these correlations - see Figure 10-and compares favorably with but is slightly less restrictive than the analytic bound from Equation (32). Figure 10 also shows the dependence of the bounds on the assumed value of $n_{0}$.

We now discuss bounds on the symmetry energy as a function of density, $S(u)$. The lower bound to $S(u)$ is just the UG bound and was already presented in Figure 2 . Regarding the upper bound, we first consider the case when $u \geqslant 1$. In this case, a conservative upper bound, $S_{\max }$, to $S(u)$ can be found for each value of $S_{0}$ by using the upper bound to $L$ for that $S_{0}$ and the upper $95.4 \%$ confidence limits for the $K_{\text {sym }}-L$ and $Q_{\text {sym }}-L$ correlations from Equations (36) and (37). We show these $S_{\max }$ bounds for several values of $S_{0}$ in Figure 11 . We also show a more realistic upper limit when the central values of the $K_{\mathrm{sym}}-L$ and $Q_{\text {sym }}-L$ correlations are used. The same procedure leads to an effective lower limit to $S(u)$ for $u \leqslant 1, S_{\min }$, for each value of $S_{0}$. We stress that this effective lower limit is not a strict lower limit but a good approximation with desirable properties: as seen in Figure 11, this procedure does not violate the UG bound, smoothly converges to the UG bound as $u \rightarrow 0$, and has the correct noninteracting limiting behavior as $u \rightarrow 0$. For $u \leqslant 1$ there is no practical upper bound to $S(u)$ because $L$ has a very small, and possibly negative, lower bound.

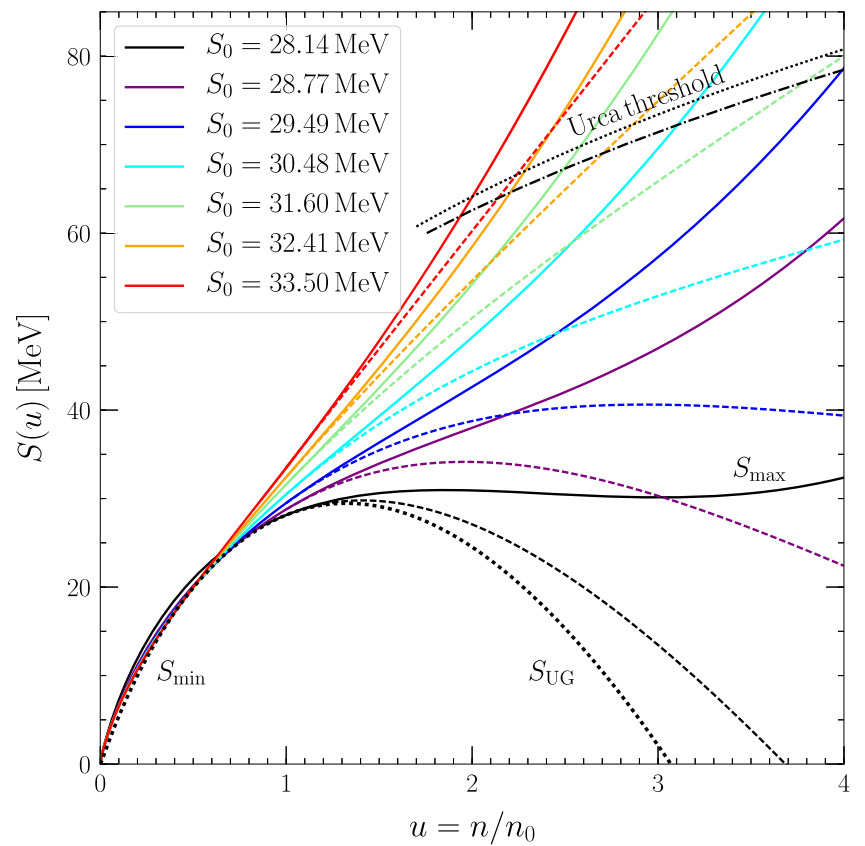

Figure 11. Upper and lower limits to the symmetry energy from the UG constraint. Each solid (dashed) curve denotes the results for different choices of $S_{0}$ and uses the upper $95.4 \%$ confidence errors (central values) of the correlations between $K_{\text {sym }}$ and $L$ and between $Q_{\text {sym }}$ and $L$.For $u \geqslant 1$, the solid curves denote $S_{\max }$, an effective upper limit to $S(u)$ as discussed in the text. For $u \leqslant 1$, the solid curves denote an effective lower limit $S_{\min }$ and approach the UG bound (lower dotted curve) as $u \rightarrow 0$. The upper dotted (dotted-dashed) curve indicates the threshold for the onset of the direct nucleon Urca process without (with) the inclusion of muons.

\subsection{Nuclear Surface Symmetry Energy}

Our UG bound has direct implications for the symmetry properties of the nuclear surface. For example, an effective maximum value of the surface symmetry energy parameter $S_{S}$ can be estimated by making the following argument. We assume a potential model for the total free energy of semiinfinite symmetric matter in which the total energy density $\mathcal{E}(u)$ is the sum of bulk and gradient contributions:

$$
\mathcal{E}(u)=u n_{0} E_{\mathrm{SNM}}(u)+Q n_{0}^{2}\left(\frac{d u}{d z}\right)^{2},
$$

where $z$ is the distance from the surface and $Q$ is a constant. Minimizing the total energy per unit surface area $\int_{-\infty}^{\infty} \mathcal{E}(u) d z$ with respect to $u(z)$ for a fixed number of baryons introduces the Lagrangian parameter $\mu$ and results in

$$
u\left(E_{\mathrm{SNM}}(u)-\mu\right)=Q n_{0}\left(\frac{d u}{d z}\right)^{2}
$$

see Ravenhall et al. (1983). One must have $\mu=E_{\mathrm{SNM}}(1)=E_{0}$ so that the gradient vanishes at the nuclear center $(u=1, z \rightarrow-\infty)$. A typical surface thickness parameter

$$
\begin{aligned}
& t_{90-10}=\int_{0.1}^{0.9} \frac{d u}{(d u / d z)} \equiv \sqrt{\frac{Q n_{0}}{T}} I_{t}, \\
& I_{t}=\int_{0.1}^{0.9} \frac{d u}{\sqrt{f_{B}(u)}}
\end{aligned}
$$




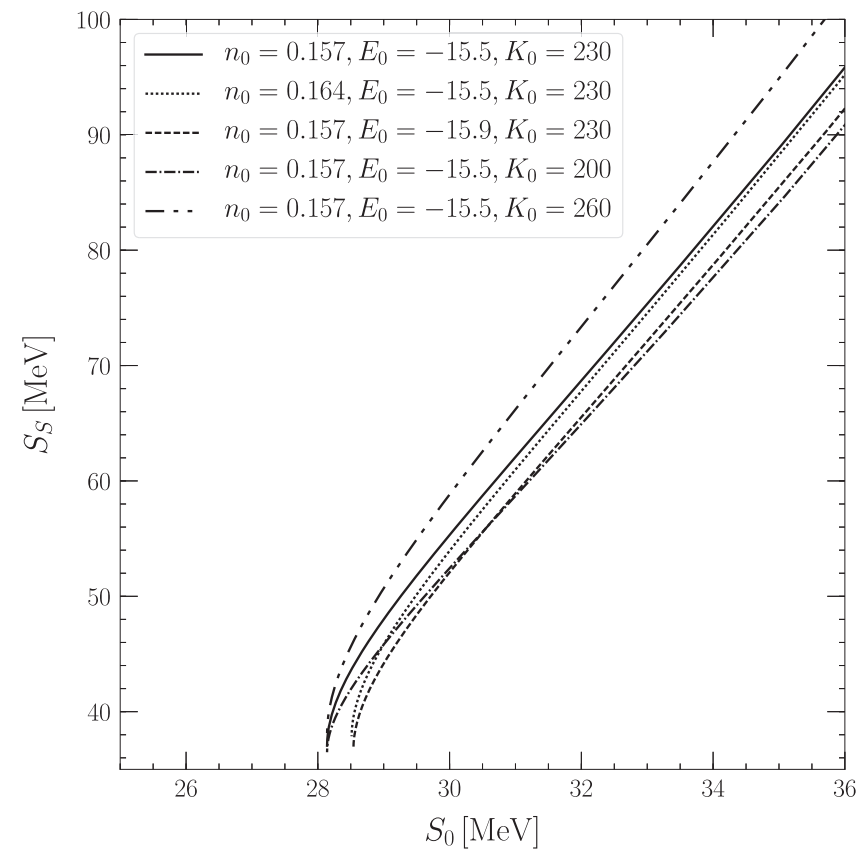

Figure 12. The maximum surface symmetry parameter $S_{S}$ as a function of $S_{0}$. The solid black curve assumes $n_{0}=0.157 \mathrm{fm}^{-3}, E_{0}=-15.5 \mathrm{MeV}$, and $K_{0}=230 \mathrm{MeV}$. Dotted (dashed) curves instead assume $n_{0}=0.164 \mathrm{fm}^{-3}$ $\left(E_{0}=-15.9 \mathrm{MeV}\right)$. The upper (lower) dashed-dotted curves assume $K_{0}=260(200) \mathrm{MeV}$.

can be defined where, phenomenologically, $t_{90-10} \simeq 2.5 \mathrm{fm}$ and

$$
\begin{aligned}
f_{B}(u) & =u \frac{E_{\mathrm{SNM}}(u)-\mu}{T} \\
& =u\left[u^{2 / 3}+a^{\prime} u+b^{\prime} u^{4 / 3}+c^{\prime} u^{5 / 3}+d^{\prime} u^{2}-\frac{E_{0}}{T}\right] .
\end{aligned}
$$

The surface tension of asymmetric matter can be expanded in terms of the neutron excess $\delta=1-2 x$ at the center of the nucleus,

$$
\sigma=\sigma_{s}-\sigma_{\delta} \delta^{2}
$$

so the surface symmetry energy is $S_{S} A^{2 / 3}=4 \pi r_{o}^{2} \sigma_{\delta} A^{2 / 3}$. It can be shown that

$$
\begin{aligned}
\sigma_{\delta} & =S_{0} n_{0} \int_{-\infty}^{\infty} u\left(\frac{S_{0}}{S(u)}-1\right) d z \equiv S_{0} n_{0} \sqrt{\frac{Q n_{0}}{T}} I_{\delta}, \\
I_{\delta} & =\int_{0}^{1} \frac{u}{\sqrt{f_{B}(u)}}\left(\frac{S_{0}}{S(u)}-1\right) d u,
\end{aligned}
$$

which can also be written as $S_{S}=4 \pi r_{o}^{2} n_{0} S_{0} t_{90-10}\left(I_{\delta} / I_{t}\right)$. Note that the integrand of $I_{\delta}$ varies as $u^{-1 / 6}$ in the limit $u \rightarrow 0$, which is why a Taylor expansion of $S$ about $u=1$ should not be used to evaluate this integral. The surface symmetry energy scales roughly as $K_{0}^{1 / 11},\left|E_{0}\right|^{-1 / 8}$, and $n_{0}^{3 / 10}$. It is clear that a maximum value of the surface symmetry parameter can be determined if the minimum allowed $S(u)$ for $u \leqslant 1$ is used in Equation (46) for each value of $S_{0}$. Figure 12 illustrates the resulting maximum value of $S_{S}$, which for $S_{0}<33 \mathrm{MeV}$ has an upper limit of about $80 \mathrm{MeV}$.

\subsection{The Crust-Core Boundary of Neutron Stars}

An approximate method of determining the location of the crust-core boundary is to consider the stability of a homogeneous fluid of nucleons in beta equilibrium. Baym et al. (1971) showed that small density fluctuations in an otherwise uniform fluid lead to instability when

$$
\mu_{p p}-\mu_{p n}^{2} \mu_{n n}^{-1}+4 \sqrt{\pi \eta \alpha \hbar c}-4 \eta \alpha\left(9 \pi n_{p}^{2}\right)^{1 / 3}=0,
$$

where $\mu_{i j}=\partial \mu_{i} / \partial n_{j}$ and $\mu_{i}$ and $n_{i}$ are the chemical potential and number density, respectively, of neutrons, protons, or electrons. In the following, we use that $\mu_{p n}=\mu_{n p}$ due to the commutativity of the derivative. The parameter $\alpha \simeq 1 / 137$ is the fine-structure constant, and $\eta$ is determined by densitygradient terms in the nuclear Hamiltonian and can be approximated by $\eta=D\left[1-4 \mu_{n p} / \mu_{n n}+\left(\mu_{n p} / \mu_{n n}\right)^{2}\right]$, where $D=4 Q / 3 \simeq 81 \mathrm{MeV} \mathrm{fm}^{5}$ (Hebeler et al. 2013). The chemical potentials and derivatives in Equation (47) are equivalent to

$$
\begin{aligned}
\mu_{n} & =\frac{\partial n E}{\partial n}-x \frac{\partial E}{\partial x}, \\
\mu_{p} & =\frac{\partial n E}{\partial n}+(1-x) \frac{\partial E}{\partial x} \\
\mu_{n n} \mu_{p p}-\mu_{n p}^{2} & =\frac{1}{n} \frac{\partial^{2} n E}{\partial n^{2}} \frac{\partial^{2} E}{\partial x^{2}}-\left(\frac{\partial^{2} E}{\partial n \partial x}\right)^{2}, \\
\mu_{n p} & =\frac{\partial^{2} n E}{\partial n^{2}}+(1-2 x) \frac{\partial^{2} E}{\partial n \partial x}-\frac{x(1-x)}{n} \frac{\partial^{2} E}{\partial x^{2}}, \\
\mu_{n n} & =\frac{\partial^{2} n E}{\partial n^{2}}-2 x \frac{\partial^{2} E}{\partial n \partial x}+\frac{x^{2}}{n} \frac{\partial^{2} E}{\partial x^{2}} .
\end{aligned}
$$

We assume a quadratic symmetry energy so that $E(u, x)=E_{\mathrm{SNM}}(u)+(1-2 x)^{2} S(u)$. The matter will be in $\beta$-equilibrum, for which $x(u)$ is determined by

$$
\mu_{n}-\mu_{p}=4 S(u)(1-2 x)=\mu_{e}=\hbar c\left(3 \pi^{2} n_{0} u x\right)^{1 / 3} .
$$

At the densities of interest for the crust-core boundary, PNM is very similar to neutron-star matter since proton fractions are of the order $2.5 \%-5 \%$.

If one employs the minimum value of $S(u)$ in the range $0<u<1$, the resulting crust-core transition density is maximized; see Figure 13. We find that the crust-core transition density decreases with an increasing $S_{0}$ and/or a decreasing $K_{0}$. The transition density is roughly proportional to the assumed value of $n_{0}$ but is insensitive to $E_{0}$ and is generally less than $0.1 \mathrm{fm}^{-3}$. This reinforces the notion that the transition density is larger than the approximate lower limit where nuclear pasta possibly exists, $u \approx 1 / 3$ (Oyamatsu \& Iida 2007).

\subsection{The Direct Urca Process}

The direct Urca process in neutron stars, in which the degenerate nucleons beta decay leading to subsequent cooling due to the loss of neutrinos and antineutrinos, requires a minimum fraction of protons, $x=1 / 9$, in the low-temperature limit and in the absence of muons due to energy and momentum conservation (Lattimer et al. 1991). Assuming again the quadratic approximation for the dependence of the energy on the neutron excess, this condition, from 


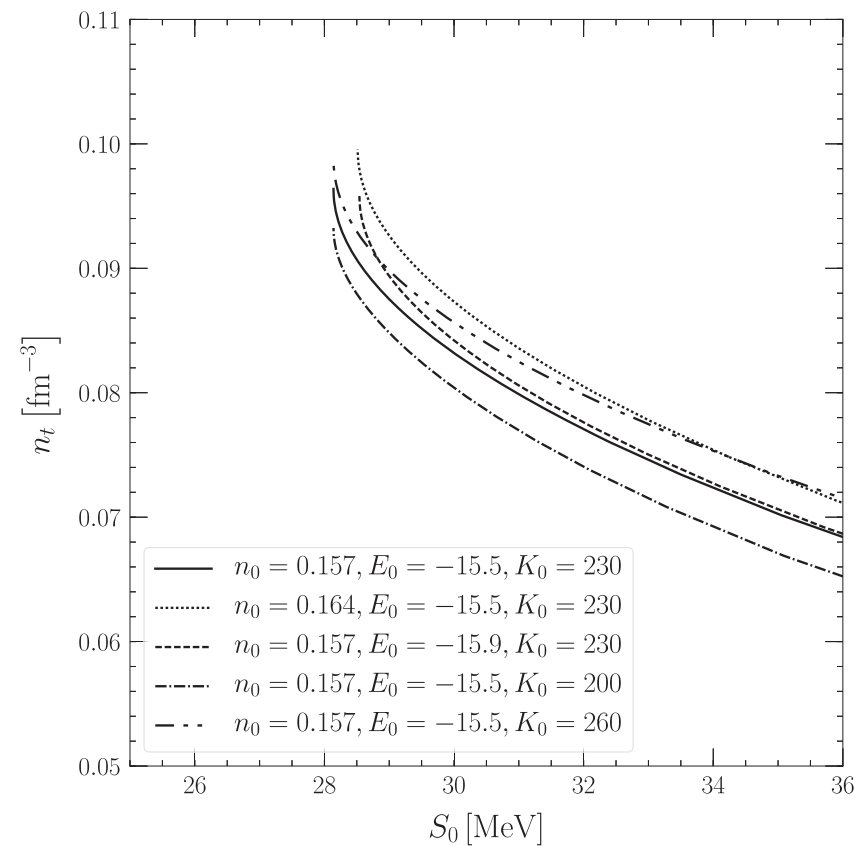

Figure 13. The maximum value of the crust-core transition density as a function of $S_{0}$. Curves are labeled as in Figure 12.

Equation (49), is simply

$$
\frac{S(u)}{u^{1 / 3}} \geqslant \frac{9}{28} \hbar c\left(\frac{\pi^{2} n_{0}}{3}\right)^{1 / 3} \simeq 51.2 \mathrm{MeV},
$$

if the presence of muons is ignored. Muons appear around the nuclear saturation density but do not change this condition significantly.

The threshold densities for the onset of the direct nucleon Urca process are shown in Figure 11, for both the conservative and realistic maximum symmetry energies implied by the UG constraint. The Urca process is always disallowed for $u \lesssim 2$ as long as $S_{0}<33.5 \mathrm{MeV}$, irrespective of assumptions concerning the maximum symmetry energy. For conservative (realistic) assumptions, and $S_{0} \lesssim 29.5$ (31.6) $\mathrm{MeV}$, the Urca threshold density is $u \gtrsim 4$, near the maximum central densities of maximum-mass stars. This means that only the highest-mass neutron stars could become capable of rapid neutrino cooling due to the Urca process. Current estimates of the temperatures and ages of observed neutron stars are consistent with the interpretation that only a small fraction have cooled rapidly (Page et al. 2004). If mass is the most important parameter controlling neutron-star thermal evolution, then this small fraction implies that only very high-mass neutron stars cool rapidly, providing empirical evidence that $S_{0} \lesssim 32 \mathrm{MeV}$.

\subsection{Neutron-star Radii and Neutron-skin Thicknesses}

The UG bounds directly impact predictions for both the neutron-star radii and the neutron-skin thickness of neutronrich nuclei, such as ${ }^{208} \mathrm{~Pb}$ and ${ }^{48} \mathrm{Ca}$. From Lattimer \& Lim (2013), the correlation between $R_{1.4}$, the radius of a $1.4 M_{\odot}$ neutron star, and the pressure of PNM at the saturation density
$\left(p_{\mathrm{PNM}}\left(n_{0}\right)\right)$ is

$$
R_{1.4} \simeq(9.52 \pm 0.49)\left(\frac{f p_{\mathrm{PNM}}\left(n_{0}\right)}{\mathrm{MeV} \mathrm{fm}^{-3}}\right)^{1 / 4} \mathrm{~km},
$$

where $f=0.94 \pm 0.02$ is a factor that corrects for the finite proton fraction of neutron-star matter. Since $p_{\mathrm{PNM}}\left(n_{0}\right)=L n_{0} / 3$, one finds, using $n_{0}=0.164 \pm 0.07 \mathrm{fm}^{-3}$, that

$$
R_{1.4} \simeq(4.51 \pm 0.26)\left(\frac{L}{\mathrm{MeV}}\right)^{1 / 4} \mathrm{~km} .
$$

With the upper limit $L \sim 80 \mathrm{MeV}$ for $S_{0} \leqslant 33 \mathrm{MeV}$, consistent with nuclear-mass measurements, we obtain $R_{1.4}<14.0 \mathrm{~km}$. The lower limit to $L, L_{0}$ for $S_{0}=S_{0}^{\mathrm{LB}}$, suggests that $R_{1.4}>9.5 \mathrm{~km}$. These limits are compatible with most estimates of neutron-star radii, but interpretations of currently proposed radius observations will probably not be significantly impacted by the UG constraint.

However, that is not the situation for the proposed neutronskin thickness experiments. Brown (2000) and Typel \& Brown (2001) found that the neutron-skin thickness $r_{n p}$ of ${ }^{208} \mathrm{~Pb}$ is related to $p_{\mathrm{PNM}}$ by

$$
r_{n p} \simeq(0.060 \pm 0.015)+0.12\left(\frac{p_{\mathrm{PNM}}\left(n=0.1 \mathrm{fm}^{-3}\right)}{\mathrm{MeV} \mathrm{fm}^{-3}}\right) \mathrm{fm} .
$$

The minimum pressure of PNM, using the constraint $E_{\mathrm{PNM}} \geqslant E_{\mathrm{UG}}$, is

$$
p_{\mathrm{PNM}, \min }(n)=\frac{2}{3} E_{\mathrm{UG}}^{0} n\left(\frac{n}{n_{0}}\right)^{2 / 3},
$$

which is independent of assumptions about the value of $n_{0}$. At the nominal density $n=0.1 \mathrm{fm}^{-3}, p_{\mathrm{PNM} \text {, min }}=0.624 \mathrm{MeV} \mathrm{fm}^{-3}$.

As previously mentioned, we cannot use either the Taylor expansions of Equations (18) and (11) or the power-law expressions of Equations (38) and (39) to estimate an upper limit to the energy or pressure of PNM because of the lack of an effective lower limit to $L$ as a function of $S_{0}$. However, the energy of PNM must be less than the Fermi-gas energy $T\left(2 n / n_{0}\right)^{2 / 3}$ because the interactions are attractive at densities less than $n_{0}$. This results in

$$
\begin{aligned}
p_{\mathrm{PNM}, \max }(n) & =n^{2} \frac{\partial E_{\mathrm{PNM}, \max }}{\partial n}=\frac{2}{3} \operatorname{Tn}\left(\frac{2 n}{n_{0}}\right)^{2 / 3} \\
& =1.701 \mathrm{MeV} \mathrm{fm}^{-3},
\end{aligned}
$$

which has been evaluated at the nominal density and is also independent of assumptions concerning $n_{0}$. One therefore finds $0.12 \mathrm{fm}<r_{n p}<0.28 \mathrm{fm}$, independent of assumptions concerning $S_{0}$. While this is consistent with most experimental results for ${ }^{208} \mathrm{~Pb}, \quad r_{n p}=0.159 \pm 0.041$ (Danielewicz \& Lee 2009) (but see Danielewicz et al. (2017), who find $\left.r_{n p}=0.223 \pm 0.018 \mathrm{fm}\right)$, the most recent neutron-skin thickness measurement from the PREX experiment (Abrahamyan et al. 2012) is $r_{n p}=0.33_{-0.18}^{+0.16} \mathrm{fm}$ and thus the mean value is larger than our upper bound. The proposed PREX-II experiment will have an estimated error in $r_{n p}$ of about $\pm 0.06 \mathrm{fm}$ 
(Horowitz et al. 2014). This should be sufficient to resolve the tension with our upper limit.

\section{Conclusions}

We presented new bounds on the symmetry energy parameters $\left(S_{0}, L\right)$ based on the conjecture that the energy of the UG is less than the energy of PNM. Specifically, we determined a minimum value for the volume symmetry parameter $S_{0}$ (equivalent to the liquid drop parameter $J$ ), $S_{0}^{\mathrm{LB}} \simeq 28.1 \mathrm{MeV}$, as well as both minimum and maximum values of the symmetry energy slope parameter $L$ for values of $S_{0} \geqslant S_{0}^{\mathrm{LB}}$. We also determined a minimum value for the symmetry incompressibility parameter $K_{\text {sym }}$ as a function of $S_{0}$. These parameters are all evaluated at nuclear saturation density $n_{0}$, but in addition, we established a minimum for the bulk symmetry energy $S(u)$ as a function of density in the vicinity of the saturation density $n_{0}$.

This conjecture is in agreement with ab initio calculations of PNM with $\mathrm{NN}$ and $3 \mathrm{~N}$ forces. Using conservative values for the Bertsch parameter $\xi_{0}$, as well as the saturation properties $E_{0}$, $n_{0}, K_{0}, K_{\text {sym }} Q_{0}$, and $Q_{\text {sym }}$ of nuclear forces, we find that symmetry energy parameter constraints from various nuclear experiments are consistent with the UG bound. However, several theoretical interactions in active use for both theoretical calculations of dense matter and tabulated equations of state used in astrophysical simulations of supernovae and neutronstar mergers violate the UG constraint. In addition, less than $52 \%$ of the more than 500 nonrelativistic potential and RMF interactions in recent compilations (Dutra et al. 2012, 2014) satisfy our constraint.

Because the conjecture establishes a maximum value of $L$ for each $S_{0}$, and because both $K_{\text {sym }}$ and the symmetry skewness parameter $Q_{\text {sym }}$ are highly correlated with $L$, a maximum symmetry energy $S(n)$ for $n \gtrsim n_{0}$ may be found for each assumed value of $S_{0}$. In addition, a minimum symmetry energy $S(n)$ for $n<n_{0}$ may also be obtained for each assumed value of $S_{0}$. Our results thus have important consequences not only for astrophysical simulations but also for nuclear structure and neutron-star structure. In particular, we obtain upper limits to the liquid-droplet surface symmetry energy parameter and the neutron-star crust-core transition density, and both upper and lower limits to the neutron-skin thickness of neutron-rich nuclei and typical neutron-star radii. We can also impose constraints on the operation of the nucleon direct Urca process in cooling neutron stars.

Obtaining experimental results from cold atoms with a finite scattering length (Hu et al. 2010; Navon et al. 2010; Horikoshi et al. 2016) will be important and may eventually offer additional insights and even more stringent constraints.

This work resulted from discussions at the YIPQS long-term and Nishinomiya-Yukawa memorial workshop on Nuclear Physics, Compact Stars, and Compact Star Mergers 2016 (YITP-T-16-02). E. E. K., J. M. L., and I. T. thank the hospitality of the YITP. The authors thank Stefano Gandolfi and other participants of the workshop as well as Sanjay Reddy and Achim Schwenk for useful discussions, Hajime Togashi for providing numerical data, and Munekazu Horikoshi and Yoji Ohashi for inspiring suggestions. This work was supported in part by NSF Grant No. PHY-1430152 (JINA Center for the Evolution of the Elements), U.S. Department of Energy Grants DE-AC02-87ER40317 and
DE-FG02-00ER41132, JSPS/MEXT KAKENHI Grant Nos. 15K05079, 15H03663, 16K05350, 24105001, 24105008, and Slovak Grant VEGA-1/0469/15.

\section{ORCID iDs}

Ingo Tews (ํ) https://orcid.org/0000-0003-2656-6355 James M. Lattimer (10 https://orcid.org/0000-0002-5907-4552 Akira Ohnishi (1) https://orcid.org/0000-0003-1513-0468 Evgeni E. Kolomeitsev (i) https://orcid.org/0000-00031160-2050

\section{References}

Abrahamyan, S., Ahmed, Z., Albataineh, H., et al. 2012, PhRvL, 108, 112502 Akmal, A., Pandharipande, V. R., \& Ravenhall, D. G. 1998, PhRvC, 58, 1804 Bauswein, A., Stergioulas, N., \& Janka, H.-T. 2016, EPJA, 52, 56 Baym, G., Bethe, H. A., \& Pethick, C. J. 1971, NuPhA, 175, 225 Blaizot, J. P. 1980, PhR, 64, 171

Brown, B. A. 2000, PhRvL, 85, 5296

Cai, B.-J., \& Chen, L.-W. 2014, arXiv:1402.4242

Carbone, A., Polls, A., Providencia, C., Rios, A., \& Vidana, I. 2014, EPJA, 50, 13

Carlson, J., Cowell, S., Morales, J., Ravenhall, D. G., \& Pandharipande, V. R. 2002, PThPS, 146, 363

Carlson, J., Gandolfi, S., \& Gezerlis, A. 2012, PTEP, 2012, 01A209

Chen, L. W., Cai, B. J., Ko, C. M., et al. 2009, PhRvC, 80, 014332

Danielewicz, P., Lacey, R., \& Lynch, W. G. 2002, Sci, 298, 1592

Danielewicz, P., \& Lee, J. 2009, NuPhA, 818, 36

Danielewicz, P., Singh, P., \& Lee, J. 2017, NuPhA, 958, 147

Drischler, C., Carbone, A., Hebeler, K., \& Schwenk, A. 2016a, PhRvC, 94, 054307

Drischler, C., Hebeler, K., \& Schwenk, A. 2016b, PhRvC, 93, 054314

Ducoin, C., Margueron, J., Providência, C., \& Vidaña, I. 2011, PhRvC, 83, 045810

Dutra, M., Loureno, O., Avancini, S. S., et al. 2014, PhRvC, 90, 055203

Dutra, M., Loureno, O., Martins, J. S. S., et al. 2012, PhRvC, 85, 035201

Dyhdalo, A., Furnstahl, R. J., Hebeler, K., \& Tews, I. 2016, PhRvC, 94, 034001

Farine, M., Pearson, J. M., \& Tondeur, F. 1997, NuPhA, 615, 135

Fischer, T., Hempel, M., Sagert, I., Suwa, Y., \& Schaffner-Bielich, J. 2014, EPJA, 50, 46

Friedman, B., \& Pandharipande, V. R. 1981, NuPhA, 361, 502

Gandolfi, S., Carlson, J., \& Reddy, S. 2012, PhRvC, 85, 032081

Gandolfi, S., Gezerlis, A., \& Carlson, J. 2015, ARNPS, 65, 303

Gezerlis, A., \& Carlson, J. 2010, PhRvC, 81, 025803

Hebeler, K., Lattimer, J. M., Pethick, C. J., \& Schwenk, A. 2010, PhRvL, 105, 161102

Hebeler, K., Lattimer, J. M., Pethick, C. J., \& Schwenk, A. 2013, ApJ, 773, 11

Hebeler, K., \& Schwenk, A. 2010, PhRvC, 82, 014314

Horikoshi, M., Koashi, M., Tajima, H., et al. 2016, arXiv:1612.04026

Horowitz, C. J., Kumar, K. S., \& Michaels, R. 2014, EPJA, 50, 48

Khan, E., Margueron, J., \& Vidaña, I. 2012, PhRvL, 109, 092501

Klähn, T., Blaschke, D., Typel, S., et al. 2006, PhRvC, 74, 035802

Kortelainen, M., Lesinski, T., Moré, J., et al. 2010, PhRvC, 82, 024313

Ku, M. J. H., Sommer, A. T., Cheuk, L. W., \& Zwierlein, M. W. 2012, Sci, 335,563

Kuhnle, E. D., Hu, H., Liu, X.-J., et al. arXiv:1001.3200

Lattimer, J. M., \& Lim, Y. 2013, ApJ, 771, 51

Lattimer, J. M., \& Prakash, M. 2001, ApJ, 550, 426

Lattimer, J. M., \& Prakash, M. 2007, PhR, 42, 109

Lattimer, J. M., Prakash, M., Pethick, C. J., \& Haensel, P. 1991, PhRvL, 66,2701

Lattimer, J. M., \& Steiner, A. W. 2014, EPJA, 50, 40

Lee, T. D., \& Yang, C. N. 1957, PhRv, 105, 1119

Lynn, J. E., Tews, I., Carlson, J., et al. 2016, PhRvL, 116, 062501

Machleidt, R., \& Slaus, I. 2001, JPhG, 27, R69

Margueron, J., Hoffmann Casali, R., \& Gulminelli, F. 2017, arXiv:1708.06894

Maslov, K. A., Kolomeitsev, E. E., \& Voskresensky, D. N. 2016, NuPhA, 950,64

Matsuo, M. 2006, PhRvC, 73, 044309

Mumpower, M. R., Surman, R., McLaughlin, G. C., \& Aprahamian, A. 2016, PrPNP, 86, 86

Navon, N., Naxcimbéne, S., Chevy, F., \& Salomon, C. 2010, Sci, 328, 729

Oertel, M., Hempel, M., Klähn, T., \& Typel, S. 2017, RvMP, 89, 015007

Oyamatsu, K., \& Iida, K. 2007, PhRvC, 75, 015801 
The Astrophysical Journal, 848:105 (15pp), 2017 October 20

Page, D., Lattimer, J. M., Prakash, M., \& Steiner, A. W. 2004, ApJS, 155, 623 Piekarewicz, J. 2010, JPhG, 37, 064038

Ravenhall, D. G., Pethick, C. J., \& Lattimer, J. M. 1983, NuPhA, 407, 571

Schwenk, A., \& Pethick, C. J. 2005, PhRvL, 95, 160401

Schwinger, J. 1947, PhRv, 72, 738

Shlomo, S., Kolomietz, V. M., \& Coló, G. 2006, EPJA, 30, 23

Stone, J. R., Stone, N. J., \& Moskowski, S. A. 2015, PhRvC, 89, 044316

Tews, I., Gandolfi, S., Gezerlis, A., \& Schwenk, A. 2016, PhRvC, 93, 024305

Tews, I., Krüger, T., Hebeler, K., \& Schwenk, A. 2013, PhRvL, 110, 032504
Togashi, H., \& Takano, M. 2013, NuPhA, 902, 53

Typel, S., \& Brown, B. A. 2001, PhRvC, 64, 27302

Vidaña, I., Providência, C., Polls, A., \& Rios, A. 2009, PhRvC, 80, 045806

Wellenhofer, C., Holt, J. W., \& Kaiser, N. 2016, PhRvC, 93, 055802

Zhang, N.-B., Cai, B.-J., Li, B.-A., Newton, W. G., \& Xu, J. 2017, arXiv:1704. 02687

Zürn, G., Lompe, T., Wenz, A. N., et al. 2013, PhRvL, 110, 135301

Zwierlein, M. W. 2015, in Novel Superfluids, Vol. 2, ed. K. H. Bennemann \& J. B. Ketterson (Oxford: Oxford Univ. Press), 269 